Cahiers de la recherche sur les droits fondamentaux

$12 \mid 2014$

Droit et psychiatrie

\title{
L'article 66 de la Constitution, le juge administratif et la protection des droits du malade mental
}

\section{Marie-Joëlle Redor-Fichot}

\section{(2) OpenEdition}

\section{Journals}

Édition électronique

URL : http://journals.openedition.org/crdf/1874

DOI : $10.4000 /$ crdf. 1874

ISSN : 2264-1246

Éditeur

Presses universitaires de Caen

Édition imprimée

Date de publication : 1 décembre 2014

Pagination : 29-45

ISBN : 978-2-84133-507-7

ISSN : 1634-8842

\section{Référence électronique}

Marie-Joëlle Redor-Fichot, «L'article 66 de la Constitution, le juge administratif et la protection des droits du malade mental », Cahiers de la recherche sur les droits fondamentaux [En ligne], 12 | 2014, mis en ligne le 01 octobre 2015, consulté le 11 février 2020. URL : http://journals.openedition.org/crdf/ 1874 ; DOI : 10.4000/crdf.1874 


\title{
L'article 66 de la Constitution, le juge administratif et la protection des droits du malade mental
}

\author{
Marie-Joëlle REDOR-FICHOT \\ Professeure de droit public à l'université de Caen Basse-Normandie \\ Directrice du Centre de recherche sur les droits fondamentaux et les évolutions du droit (CRDFED, EA 2132)
}

I. Les incertitudes de la protection offerte par les juges aux droits des patients

A. Le champ incertain de la compétence du juge administratif

1. Les contestations relatives aux mesures individuelles prises à l'égard du patient en soins sans consentement

2. Les actions en réparation des dommages causés au patient

B. Une protection affaiblie par les réticences des juges à statuer sur certaines questions

1. Traitement médical et programme de soins

2. Sorties d'essai et permissions de sortie

3. Placement en unité pour malades difficiles (UMD)

II. Un système de protection à repenser?

A. Les fondements contestables de la répartition actuelle

1. La combinaison problématique de principes contradictoires

2. Des fondements dépassés

B. Une nouvelle répartition?

1. L'option de la concurrence

2. L'option du bloc de compétence

En confiant à l'autorité judiciaire le soin de protéger l'individu contre les détentions arbitraires, l'article 66 a inscrit dans la Constitution de 1958 une tradition née au XIX siècle selon laquelle le juge judiciaire est un meilleur défenseur des libertés que ne pourrait l'être le juge administratif. Dès 1838, dans l'objectif de préserver l'individu des abus de pouvoir de l'administration, la loi Esquirol avait ainsi confié au juge judiciaire, plutôt qu'au juge administratif soupçonné de partialité en faveur de l'administration, le soin de statuer sur le bien-fondé des internements psychia- triques $^{1}$. Mais la compétence judiciaire se heurtait à la loi des 16 et 24 août 1790 qui interdit aux tribunaux judiciaires de faire obstruction aux décisions de l'administration. Se fondant sur ce principe de séparation des autorités administratives et judiciaires, le Tribunal des conflits a décidé en $1946^{2}$ de cantonner la compétence judiciaire au seul examen de la légalité interne de la décision d'internement, réservant au juge administratif l'examen de sa régularité c'est-à-dire de sa légalité externe (respect des règles de compétence, de forme et de procédure). Le requérant

1. Loi $\mathrm{n}^{\circ} 7443$ sur les aliénés du 30 juin 1838 .

2. TC, Sieur Machinot c. Préfet de police, 6 avril 1946, Recueil Lebon, p. 326. 
devait donc intenter deux actions devant deux juridictions différentes s'il voulait contester tous les éléments de la décision d'internement. Jusqu'à 1997, le Tribunal des conflits imposait également au requérant d'intenter deux actions différentes pour obtenir réparation du dommage causé d'une part par l'illégalité interne (devant le juge judiciaire), d'autre part de celui causé par l'illégalité externe (devant le juge administratif) ${ }^{3}$. Autant dire qu'une telle jurisprudence était davantage arcboutée sur la préservation de la compétence du juge administratif, que tournée vers la défense des droits du requérant. Le revirement opéré en $1997^{4}$ a permis d'améliorer sensiblement les choses en unifiant au profit du juge judiciaire le contentieux de la réparation du dommage causé par une décision d'internement illégale (désormais une seule action intentée devant le juge judiciaire permettait donc au requérant d'obtenir réparation du dommage subi quelle que soit l'origine de l'illégalité commise); mais ce revirement était seulement partiel puisque le juge judiciaire devait surseoir à statuer en attendant la réponse du juge administratif sur l'illégalité externe de la décision contestée.

En 2010, la Cour européenne des droits de l'homme a condamné la France pour le manque d'effectivité des recours susceptibles d'être intentés par le patient et ses proches en cas d'internement ${ }^{5}$. Le législateur de $2011 \mathrm{a}$ donc simplifié la répartition des compétences et opté pour une unification du contentieux au profit du juge judiciaire $^{6}$. Selon le Code de la santé publique, en effet ${ }^{7}$, pour les recours introduits à compter du $1^{\mathrm{er}}$ janvier 2013, le juge judiciaire est seul compétent pour statuer sur la régularité des décisions administratives d'admission en soins sans consentement. Ainsi, lorsque le juge des libertés et de la détention (JLD) se prononce au titre de l'article L. 3211-12 sur la demande de mainlevée de la mesure de soins psychiatriques sans consentement quelle qu'en soit la forme, ou lorsqu'il vérifie la nécessité du maintien de l'hospitalisation complète dans le cadre de sa saisine imposée par l'article L. 3211-12-1, il peut apprécier la légalité tant externe qu'interne de la décision qui lui est soumise. De même le tribunal de grande instance peut-il désormais apprécier lui-même la régularité de la mesure de soins sans consentement lorsqu'il statue sur la réparation du dommage causé au patient par la décision.

Le juge administratif n'intervient donc plus sur la régularité de la décision d'admission en soins sans consentement ${ }^{8}$ : que ce soit pour demander la mainlevée de la mesure ou pour obtenir réparation du préjudice découlant de son illégalité, seul le juge judiciaire est désormais compétent sans même qu'il soit nécessaire de poser une question préjudicielle au juge administratif 9 . Mais l'unification du contentieux opérée par la loi de 2011 est loin d'être complète, laissant ouverte la question de savoir ce qui relève encore de la compétence du juge administratif ${ }^{10}$.

Faut-il alors modifier l'article 66 de la Constitution? Quoiqu'elle puisse sembler iconoclaste, la question mérite d'être posée au vu des difficultés que continue à susciter

3. TC, Préfet de Paris, 27 novembre 1995, nº 2973 : «si l’autorité judiciaire est seule compétente, en vertu des articles L. 333 et suivants du code de la santé publique, pour apprécier la nécessité d'une mesure de placement d'office en hôpital psychiatrique et les conséquences qui peuvent en résulter, il appartient à la juridiction administrative d'apprécier la régularité de la décision administrative qui ordonne le placement, et, le cas échéant, les conséquences dommageables de son défaut de notification ainsi que des fautes du service public qui auraient pu être commises à cet égard ».

4. Voir TC, Préfet de la région Île-de-France, 17 février 1997, nº 03045, La semaine juridique, éd. G, $\mathrm{n}^{\circ} 30,23$ juillet 1997, II, 22885, concl. J. SainteRose; Revue trimestrielle de droit civil, 1998, p. 72, obs. J. Hauser; ibid., p. 181, obs. J. Normand. Selon le Tribunal des conflits, «si l'autorité judiciaire est seule compétente, en vertu des articles L. 333 et suivants du code de la santé publique, pour apprécier la nécessité d'une mesure de placement d'office en hôpital psychiatrique et les conséquences qui peuvent en résulter, il appartient à la juridiction administrative d'apprécier la régularité de la décision administrative qui ordonne le placement; que lorsque cette dernière s'est prononcée sur ce point, l'autorité judiciaire est compétente pour statuer sur les conséquences dommageables de l'ensemble des irrégularités entachant la mesure de placement d'office».

5. Cour EDH, Baudoin c. France, 18 novembre 2010, nº 35935/03. Dans cet arrêt, la France est condamnée par la Cour notamment pour violation de l'article 5, $\$ 4$ de la Convention car, du fait du système français de répartition des compétences juridictionnelles, «le requérant n’a disposé d’aucun recours effectif qui lui aurait permis d'obtenir une décision judiciaire constatant l'irrégularité fondant son internement et mettant fin, par voie de conséquence, à sa privation de liberté irrégulière» (\$109). Voir J. Hauser, «Hospitalisation psychiatrique sans consentement: branle-bas de combat! », Revue trimestrielle de droit civil, 2011, p. 101-103.

6. Loi $\mathrm{n}^{\mathrm{o}}$ 2011-803 du 5 juillet 2011 relative aux droits et à la protection des personnes faisant l'objet de soins psychiatriques et aux modalités de leur prise en charge.

7. Art. L. 3216-1 du Code de la santé publique (CSP): «La régularité des décisions administratives prises en application des chapitres II à IV du présent titre ne peut être contestée que devant le juge judiciaire./Le juge des libertés et de la détention connaît des contestations mentionnées au premier alinéa du présent article dans le cadre des instances introduites en application des articles L. 3211-12 et L. 3211-12-1. Dans ce cas, l'irrégularité affectant une décision administrative mentionnée au premier alinéa du présent article n'entraîne la mainlevée de la mesure que s'il en est résulté une atteinte aux droits de la personne qui en faisait l'objet./Lorsque le tribunal de grande instance statue sur les demandes en réparation des conséquences dommageables résultant pour l'intéressé des décisions administratives mentionnées au premier alinéa, il peut, à cette fin, connaître des irrégularités dont ces dernières seraient entachées».

8. Le Conseil d'État avait cependant développé une jurisprudence protectrice des droits du patient en matière de motivation obligatoire et de respect du principe du contradictoire; voir CE, 9 novembre 2001, nº 235247 et CE, 27 mai 2011, n 330267. De même le Conseil d'État avait-il admis dans une ordonnance du $1^{\mathrm{er}}$ avril 2010 que le juge administratif puisse lever la mesure d'internement en cas d'irrégularité suffisamment grave dans le cadre d'un référé-liberté (req. $\mathrm{n}^{\circ} 335753$ ).

9. On peut remarquer que cette réforme s'inscrit dans un contexte favorable à l'élargissement de la compétence judiciaire en matière de questions accessoires (voir notamment TC, SCEA du Chéneau, 17 octobre 2011, $\mathrm{n}^{\circ} \mathrm{C}_{3} 828$ et TC, SNC Green Yellow, 12 décembre 2011, $\mathrm{n}^{\circ}$ C3841).

10. Voir notamment C. Castaing, "La volonté des personnes admises en soins psychiatriques sans consentement. Quel droit pour quel juge?», L'actualité juridique. Droit administratif, no 3 , 2013, p. 153-184; A. Farinetti, "L'unification du contentieux des soins psychiatriques sans consentement par la loi du 5 juillet 2011 ", Revue de droit sanitaire et social, n 1, janvier-février 2012, p. 111-120; A. Pena, "Internement psychiatrique, liberté individuelle et dualisme juridictionnel: la nouvelle donne », Revue française de droit administratif, ${ }^{\circ}{ }_{5}, 2011$, p. 951-966; M. Vialettes, M. Grosset, "L'unification du contentieux de l'hospitalisation sans consentement. De quelques questions posées par sa mise en œuvre au $1^{\text {er }}$ janvier 2013", La semaine juridique, éd. G, nº 6, 4 février 2013, doctr. nº 157. 
le contrôle juridictionnel des soins psychiatriques sans consentement. Les incertitudes relatives à la répartition des compétences juridictionnelles entraînent en effet des conséquences potentiellement problématiques pour la protection des droits des patients (I), ce qui implique de s'interroger sur la pertinence des fondements d'un tel système de protection (II).

\section{Les incertitudes de la protection offerte par les juges aux droits des patients}

L'étude de la jurisprudence relative aux patients en soins psychiatriques sans consentement révèle des hésitations et des contradictions qui conduisent l'observateur à une certaine perplexité. Au sein d'un même ordre de juridiction en effet, les arrêts rendus paraissent souvent contradictoires sans que se dessine une véritable cohérence. La protection des droits des patients pourrait ainsi se trouver affaiblie par les incertitudes relatives à ce qui subsiste de la compétence du juge administratif (A) et par les réticences des juges à statuer sur certains contentieux (B).

\section{A. Le champ incertain de la compétence du juge administratif}

Avant la réforme de 2011, la jurisprudence était déjà très incertaine quant à la répartition des compétences entre les deux ordres: ces incertitudes résultaient d'arrêts contradictoires concernant à la fois les contestations relatives aux droits des patients ${ }^{11}$, celles relatives au transfert d'établissement et au placement en unité pour malades difficiles $(\mathrm{UMD})^{12}$, ou encore aux sorties d'essai ${ }^{13}$.

Or ces incertitudes ne sont pas levées par la réforme de 2011, bien au contraire. L'article L. 3216-1 du CSP se contente en effet de donner compétence au juge judiciaire pour statuer sur la légalité tant externe qu'interne des décisions prises en application des chapitres II à IV du titre II, c'est-à-dire des décisions individuelles relatives à l'admission et au maintien du patient en soins sans consentement, quelle qu'en soit la forme, ainsi que pour statuer sur la réparation des dommages résultant pour le patient de ces décisions ${ }^{14}$. On peut en déduire que le juge administratif reste compétent pour statuer sur les litiges relatifs aux patients en hospitalisation ou en soins ambulatoires libres; il est également compétent pour statuer sur la légalité des actes réglementaires édictés dans le cadre des soins sans consentement ${ }^{15}$. Au-delà cependant commencent les incertitudes quant au champ exact de sa compétence ${ }^{16}$.

Quoique les recours contre les mesures prises au titre du chapitre I semblent exclus de la compétence judiciaire, la jurisprudence est en réalité très loin d'être claire, tandis que d'autres décisions relevant des chapitres II à IV posent elles aussi problème (1). Quant aux actions en réparation, la formulation «les demandes en réparation des conséquences dommageables résultant pour l'intéressé de [ces] décisions administratives » est susceptible de diverses interprétations et laisse ouverte une possible extension de la compétence judiciaire (2).

\section{Les contestations relatives aux mesures individuelles prises à l'égard du patient en soins sans consentement}

La formulation de l'article L. 3216-1 laisse penser que la contestation des décisions qui sont prises dans le cadre du chapitre I consacré aux droits des personnes faisant l'objet de soins psychiatriques relève de la seule compétence du juge administratif, notamment celles qui feraient obstacle à l'exercice de ces droits durant la période pendant laquelle

11. Sur les restrictions à la correspondance, voir CE, ord., 15 mai 2002, no 239487: compétence administrative; CE, Hittler, 4 octobre 1967: compétence administrative; CAA Paris, 19 mai 2005, nº 03 PA01855: compétence judiciaire; C. Landais, «Les limites du bloc de compétence judiciaire en matière d'hospitalisation d'office", conclusions sur CE, 6 avril 2007, $\mathrm{n}^{\circ} 280494$, L'actualité juridique. Droit administratif, $\mathrm{n}^{\circ}$ 7, 2008, p. 416-419: compétence administrative. Concernant les restrictions au droit à l'information du patient sur ses droits, voir CAA Paris, 2 juin $2005, n^{\circ} 04 \mathrm{PA0} 3714$ : compétence judiciaire; sur les restrictions aux droits de visite, voir CE, ord., 3 mars 2003, $\mathrm{n}^{\circ}$ 254625: compétence judiciaire.

12. Sur le placement en UMD, voir CE, sect., E. A., 28 juillet 2000, $\mathrm{n}^{\circ} 151068$ : compétence administrative; CE, ord., 3 mars 2003 , $\mathrm{n}^{\circ} 254625$ : compétence administrative; en revanche CE, ord., 14 octobre 2004, n 273047, mentionné aux tables du Recueil Lebon: compétence judiciaire car le placement en UMD est le corollaire du placement d'office.

13. TC, 27 novembre 1995, nº 02973 : compétence judiciaire; CE, 17 novembre 1997, n 170531 : compétence administrative mais recours irrecevable; CE, 24 septembre 2010, $n^{\circ}$ 329628, mentionné aux tables du Recueil Lebon: compétence administrative et recours recevable contre les autorisations de sortie d'essai; TA Rennes, juge des référés, $M^{m e} A$ c. CHS Guillaume Régnier, 18 juin 2012, nº 1202373: compétence judiciaire.

14. Décisions sur lesquelles le JLD est conduit à statuer dans le cadre de son contrôle systématique (pour les hospitalisations complètes) et dans le cadre des demandes de mainlevée (y compris pour les soins ambulatoires sans consentement), ainsi qu'à l'occasion des actions en réparation des préjudices causés au patient.

15. Voir CAA Bordeaux, 6 novembre 2012, n 11BXo179o, L'actualité juridique. Droit administratif, n² 21, 2013, p. 115-119, concl. D. Katz, qui annule pour atteinte excessive au droit au respect de la vie privée le règlement de l'hôpital interdisant de manière générale et absolue les relations sexuelles entre patients; CE, 20 novembre 2009, $\mathrm{n}^{\circ} 313598$ : annulation du refus du préfet d'inscrire le droit à un avocat dans la charte d'accueil de l'infirmerie psychiatrique de la préfecture de police de Paris, L'actualité juridique. Droit pénal, nº 4, 2010, p. 197, note É. Péchillon. Voir également CE, Aubrun et autres c. Groupe hospitalier de Villejuif, 28 janvier 1955, Recueil Lebon, p. 50, à propos des activités religieuses des patients mais cet arrêt ne concernait pas spécifiquement les malades internés.

16. Selon Éric Péchillon, le juge administratif reste logiquement compétent pour toutes les mesures relatives au fonctionnement interne des hôpitaux, toutes les décisions réglementaires et individuelles affectant les usagers, ainsi que pour les actions en responsabilité, hors le cas des dommages causés par l'illégalité de la décision d'admission ou de maintien en soins psychiatriques sans consentement; voir É. Péchillon, «Le pouvoir médical face au refus de consentement: un savant dosage effectué par le juge des référés », note sous TA Rennes, $M^{m e}$ A c. CHS Guillaume Régnier, 18 juin 2012, $\mathrm{n}^{\circ} 1202373$, La semaine juridique, édition administrations et collectivités territoriales, $\mathrm{n}^{\circ} 4 \mathrm{O}, 8$ octobre 2012, chron. $\mathrm{n}^{\circ} 2321$. 
la personne fait l'objet de soins sans son consentement, tels par exemple que le droit d'émettre et recevoir des courriers $^{17}$, ou de se livrer aux activités religieuses et philosophiques de son choix. L'article L. 3211-3, inscrit au chapitre I, dispose notamment que «les restrictions à l'exercice de [ses] libertés individuelles [du patient en soins psychiatriques sans consentement] doivent être adaptées, nécessaires et proportionnées à son état mental et à la mise en œuvre du traitement requis». L'appréciation de la légalité de ces restrictions devrait donc logiquement relever du juge administratif ${ }^{18}$, soit à l'occasion d'un recours pour excès de pouvoir exercé contre ces restrictions, soit par le biais d'un référé-liberté en demandant la suspension. Ce qui est en cause en effet dans ces décisions ne concerne pas directement la liberté individuelle au sens de l'article 66 (droit à la sûreté du patient), mais d'autres libertés dont la protection ne relève pas en principe du juge judiciaire.

Pourtant, si le juge administratif est bien compétent pour statuer sur la légalité de ces mesures lorsqu'il s'agit pour l'administration de les réglementer de manière générale, on s'aperçoit que les solutions sont plus incertaines s'agissant des décisions individuelles prises en la matière à l'encontre du patient. Il en va ainsi notamment lorsque les restrictions aux droits du patient sont fondées sur la nécessité médicale. Il arrive en effet que le juge administratif les considère comme étroitement liées aux décisions d'admission ou de maintien en soins sans consentement et qu'il s'estime dès lors incompétent pour en connaître. Antérieurement à la réforme, le Conseil d'État saisi d'un référé-liberté a par exemple jugé que la contestation des restrictions aux visites conduit le juge à apprécier le bienfondé du régime d'hospitalisation ${ }^{19}$, ce qui relève du seul juge judiciaire. En pratique aujourd'hui, c'est généralement à la faveur de l'examen par le juge judiciaire de la mesure de soins sans consentement ou d'une action en réparation portée devant lui que le requérant conteste les restrictions affectant son droit à l'information, ses droits de visite, de correspondance. Dans le cadre du contrôle systématique qu'il doit désormais opérer, le JLD a ainsi examiné l'atteinte aux droits du patient de communiquer avec l'extérieur ${ }^{20}$, alors que la jurisprudence administrative antérieure avait estimé, après des hésitations, que la contestation des restrictions à la correspondance du patient relève du juge administratif ${ }^{21}$. En revanche, si le patient ne souhaite pas contester la décision de soins sans consentement, mais seulement ses modalités d'application, c'est au juge administratif qu'il devrait logiquement s'adresser. Encore faut-il, pour pouvoir en contester la légalité, que la situation puisse être qualifiée de décision, ce qui n'est pas le cas des agissements et des abstentions ${ }^{22}$. Resterait alors la procédure du référé-liberté mais dont les conditions draconiennes laissent peu d'espoir de succès. En outre, le juge administratif estime généralement que certaines restrictions aux droits du patient ou de

17. Pour un exemple de compétence du juge administratif sur une mesure individuelle de restrictions au droit à la communication et à la correspondance, avant l'entrée en vigueur de la réforme de 2011, voir CE, ord., 15 mai 2002, n 239487 . Voir également C. Landais, «Les limites du bloc de compétence judiciaire...", conclusions sur CE, 6 avril 2007, $\mathrm{n}^{\circ} 280494$.

18. Contra M. Primevert, "Le contrôle du juge sur les soins psychiatriques sans consentement en questions", La semaine juridique, éd. G, n 22 , 27 mai 2013, prat. n 625: «le JLD doit vérifier que les atteintes [au droit de consentir aux soins et à la liberté d'aller et de venir] sont "adaptées, nécessaires et proportionnées aux objectifs poursuivis” (Cons. const., déc. 26 nov. 2010, n 2010-71 QPC) et ce par un contrôle précis et motivé, dans chaque cas». Or, si ce contrôle est effectué par le JLD lors de sa saisine systématique, on ne voit pas pourquoi il ne l'effectuerait pas également en cas de saisine d'une demande de mainlevée ou de réparation du dommage résultant de la décision d'admission en soins sans consentement.

19. CE, ord., 3 mars 2003, n 254625: «Sur les conclusions tendant à ce qu'il soit ordonné au directeur de l'établissement public de santé de SaintAndré lez Lille de mettre fin, après le retour de M. Y dans cet établissement, aux contraintes thérapeutiques et aux restrictions de visite dont était assorti, jusqu'à son transfert, le régime d'hospitalisation de l'intéressé: Considérant qu'il n'appartient qu'au juge judiciaire, qui a d'ailleurs été saisi par les intéressés, de connaître de la contestation soulevée par les requérants, qui porte sur le bien-fondé du régime auquel est soumis M. Y, au regard de son état mental, à la suite de son hospitalisation d'office». La CAA de Bordeaux avait au contraire retenu la compétence des juridictions administratives pour statuer sur la méconnaissance par l'établissement psychiatrique du droit au respect des correspondances du patient en placement d'office, et pour indemniser le tiers auquel étaient adressés les courriers (CAA Bordeaux, 27 novembre 1995, n 94 BXo1716).

20. CA Rouen, 13 octobre 2011, $\mathrm{n}^{\circ}$ 11/04627: «L'article L. 3211-3 du code de la santé publique prévoit qu'en tout état de cause, la personne qui fait l'objet de soins psychiatriques dispose du droit de saisir la commission prévue à l'article L. 3222-5 (commission départementale des hospitalisations psychiatriques), de prendre conseil d'un avocat de son choix ainsi que d'émettre ou de recevoir des courriers. Or, Madame A. se contente d'affirmer, sans l'établir d'aucune manière, n'avoir eu à sa disposition ni papier et stylo, ni moyen de communication avec l'extérieur durant les 72 premières heures de son hospitalisation".

21. Voir notamment CE, ord., 15 mai 2002, n 239487 et surtout CE, 6 avril 2007, no 280494

22. Ainsi, et de façon constante, le juge administratif s'est-il toujours refusé à statuer sur le défaut d'information du patient après la prise de décision (obligations imposées par l'article L. 3211-3 figurant au chapitre I), il renvoyait le requérant à se pourvoir auprès du juge judiciaire à l'occasion d'une action en réparation; voir CE, sect., E. A., 28 juillet 2000, $\mathrm{n}^{\circ}$ 151068. De même, pour un litige engagé avant le $1^{\mathrm{er}}$ janvier 2013 , CAA Paris, 28 janvier 2013, n⿳ 11PA01689: «lorsqu'elle prend une mesure d’hospitalisation sans consentement, l'autorité compétente doit, une fois la décision prise, informer le plus rapidement possible l'intéressé des motifs de cette mesure, de sa situation juridique et de ses droits, d'une manière appropriée à son état; [...] toutefois, l'accomplissement de cette obligation, qui se rapporte à l'exécution de la mesure d'hospitalisation et engage, le cas échéant, la responsabilité de l'établissement hospitalier devant le juge judiciaire, est sans influence sur la légalité de la mesure; [...] par suite, la circonstance que M. A... aurait reçu ces informations alors qu'il était sous l'influence de puissants sédatifs, et n'aurait notamment pas été informé de son droit à se faire assister par un avocat, ne peut être utilement invoquée devant le juge de l'excès de pouvoir». Voir également CE, 13 mars 2013, n 354976, mentionné aux tables du Recueil Lebon. Il en va de même sous l'empire de la nouvelle répartition: voir CA Nancy, 8 juillet 2013, $\mathrm{n}^{\circ}$ 13/01934, saisie d'une demande de mainlevée d'une décision préfectorale d'hospitalisation complète, la CA prononce la mainlevée de l'hospitalisation du fait du non-respect des mesures d'information du patient: «Monsieur Pascal B. n’a pas été régulièrement informé des droits dont il dispose aux termes des dispositions susvisées; [...] il y a donc lieu de constater que les arrêtés des 15 et 18 juin 2013 sont frappés de nullité, de constater l'irrégularité de la procédure issue de ces décisions, et en conséquence d'ordonner la mainlevée de la mesure d'hospitalisation ordonnée à l'encontre de Monsieur Pascal B ». 
ses proches sont intimement liées à la décision de soins sans consentement, impliquant par là même une lecture extensive de l'article L. 3216-1 ${ }^{23}$.

Les sorties d'essai de courte durée dans le cadre du régime d'hospitalisation complète sont elles aussi mentionnées au chapitre $\mathrm{I}^{24}$. La jurisprudence administrative antérieure à la réforme semble s'être stabilisée sur l'attribution au juge administratif de la contestation des mesures relatives à ces sorties d'essai, là encore après plusieurs années d'hésitations ${ }^{25}$. La question de la compétence juridictionnelle pourrait faire cependant problème aujourd'hui, notamment en ce qui concerne les permissions de sortie autorisées dans le cadre du régime de soins ambulatoires sans consentement, car les décisions mettant fin à ces permissions ont pour effet de réintégrer le patient en hospitalisation complète, ce qui implique de saisir le JLD pour qu'il statue sur le changement de régime imposé au patient. En revanche, le nombre de permissions de sortie accordées a trait au contenu du programme de soins dont nous verrons que les juges hésitent à le contrôler.

La contestation des décisions relatives aux droits garantis par le chapitre I peut donc prêter à discussion quant à la détermination du juge compétent. Il en va de même pour certaines décisions relevant des chapitres II à IV; la question est en effet de savoir ce qu'il faut entendre exactement par décisions administratives prises en application des chapitres II à $I V^{26}$.

C'est le cas notamment des décisions administratives mettant fin aux mesures de soins sans consentement. La compétence du juge administratif en la matière n'a été clairement admise dans la jurisprudence antérieure à la réforme qu'à la suite d'une décision du Tribunal des conflits rendue en $2006^{27}$. Or aujourd'hui, ces décisions sont mentionnées dans les chapitres II à $\mathrm{IV}^{28}$ et font donc partie, si on s'en tient à la lettre de l'article L. 3216-1, du champ de compétence réservé au juge judiciaire ${ }^{29}$. Pourtant ces décisions ne mettent pas en cause la liberté individuelle du patient au sens de l'article 66 de la Constitution, liberté individuelle qui justifie logiquement la compétence judiciaire. En outre, l'alinéa 2 de l'article L. 3216-1 peut être lu comme limitant la compétence judiciaire à trois hypothèses: le cas où le juge exerce son contrôle systématique sur les hospitalisations complètes, celui où il statue sur les recours visant à la mainlevée de la décision d'admission ou de maintien en soins sans consentement, celui enfin où il est saisi d'une action en réparation des conséquences dommageables résultant pour l'intéressé de ces décisions. On le voit, aucune de ces actions n'est susceptible de correspondre à la contestation d'une décision de mainlevée. Le juge administratif devrait donc être compétent sur ce point, notamment sur saisine d'un tiers craignant pour sa sécurité ou celle du malade. De manière similaire, le juge administratif a admis, avant la réforme, de statuer à la demande d'un tiers sur l'autorisation de sortie d'essai accordée à un patient ${ }^{30}$.

On peut également s'interroger sur le sort des recours visant à contester la décision de transférer un malade dans une $\mathrm{UMD}^{31}$. Ces transferts sont décidés par le préfet pour des patients faisant déjà l'objet d'un placement d'office (devenu aujourd'hui «ADRE»). Dans un arrêt de section du 28 juillet 2000, le Conseil d'État s'était reconnu compétent pour statuer sur la légalité externe et interne de la décision préfectorale transférant un patient en placement d'office dans un autre établissement pourvu d'une $\mathrm{UMD}^{32}$. Mais le juge des référés du Conseil d'État a estimé en 2004 que la décision de transférer le patient interné

23. Voir pour un litige antérieur à la réforme et concernant le refus opposé à un père de rendre visite à son fils interné: CAA Bordeaux, 11 février 2014, $\mathrm{n}^{\circ}$ 12BX02532, Gazette du palais, février 2014: «Considérant [...] qu'il appartient, en revanche, à la seule autorité judiciaire de se prononcer sur le bien-fondé des mesures qui ne sont pas détachables des soins donnés dans ce cadre; que la répartition des compétences ainsi assurée entre les deux ordres de juridiction satisfait au droit à un recours effectif garanti par la convention européenne de sauvegarde des droits de l'homme et des libertés fondamentales».

24. Voir art. L. 3211-11-1.

25. Voir CE, 24 septembre 2010, $\mathrm{n}^{\circ} 329628$ : compétence administrative pour la contestation de la légalité d'une autorisation de sortie d'essai; voir également CE, 4 octobre 2013, $\mathrm{n}^{\circ} 348858$, pour la contestation de la décision mettant fin à une sortie d'essai en 1967. Contra TC, 27 novembre 1995, n 02973: compétence judiciaire pour statuer sur la légalité des sorties d'essai («les irrégularités invoquées en ce qui concerne l'absence de décision pendant le premier semestre 1987, et les sorties à l'essai relèvent exclusivement de l'autorité judiciaire»); comparer CE, CHS d'Erstein, 17 novembre 1997, nº 170531, mentionné aux tables du Recueil Lebon: le Conseil admet la compétence de la juridiction administrative pour statuer sur la légalité d'une sortie d'essai, mais estime qu'il s'agit d'une «mesure relevant du traitement du malade hospitalisé et [qui] ne constitue pas une décision susceptible de faire l'objet d'un recours pour excès de pouvoir»; de même, CAA Douai, 12 juillet 2001, nº 98DAoo428.

26. Décisions sur lesquelles le JLD est conduit à statuer dans le cadre de son contrôle systématique (pour les hospitalisations complètes) et dans le cadre des demandes de mainlevée (y compris pour les soins ambulatoires sans consentement), ainsi qu'à l'occasion des actions en réparation des préjudices causés au patient.

27. TC, Président du Conseil de Paris c. Préfet de police de Paris, 26 juin 2006, n ${ }^{\circ} \mathrm{C}_{3513}$, Recueil Lebon, p. 634.

28. Voir les art. L. 3212-8 et L. 3213-9 CSP; voir également le cas des décisions d'hospitalisation de détenus faisant l'objet de soins psychiatriques avec leur consentement visées par l'article L. 3214-1 et celui des restrictions aux droits de ces patients énoncés dans le CSP, restrictions envisagées à l'article L. 3214-2.

29. Voir en ce sens A. Farinetti, «L'unification du contentieux...».

30. Voir CE, 24 septembre 2010, $n^{\circ}$ 329628, qui admet la recevabilité du recours pour excès de pouvoir contre l'autorisation de sortie. Comparer CE, 17 novembre 1997, $n^{\circ} 170531$ dans laquelle le Conseil s'estime compétent pour statuer, mais rejette le recours comme irrecevable; de même, CAA Douai, 12 juillet 2001, n 98DAoo428. En revanche, le Tribunal des conflits avait retenu la compétence du juge judiciaire pour statuer sur les sorties d'essai dans le cadre d'une action en réparation fondée sur divers préjudices (TC, 27 novembre 1995, nº 02973) avec une argumentation qui laisse penser que l'appréciation de leur légalité relève de celle de la nécessité de la mesure d’internement. Voir, dans le même sens, TA Nantes, 11 avril 1991, mentionné aux tables du Recueil Lebon.

31. Voir les art. L. 3222-3, R. 3222-1 sq. CSP, ces articles figurent dans le titre II du livre II du CSP.

32. CE, sect., E. A., 28 juillet $2000, \mathrm{n}^{\circ} 151068$. Voir, dans le même sens, CE, ord., 3 mars 2003 , nº 254625. 
d'office dans un établissement pourvu d'une UMD est le corollaire de la décision de placement d'office; il s'ensuit que sa contestation relève de la compétence du juge judiciaire ${ }^{33}$. Il faudrait donc en déduire qu'aujourd'hui la contestation de ces décisions relève en totalité du juge judiciaire. Pourtant, un tel transfert peut affecter des libertés du patient, autres que sa liberté individuelle, telles que son droit à une vie familiale en cas d'éloignement de sa famille. Le juge administratif ne devrait-il pas alors statuer sur le transfert ${ }^{34}$ ?

\section{Les actions en réparation des dommages causés au patient}

Selon la doctrine ${ }^{35}$, la frontière en matière de réparation des préjudices causés au patient passe par la distinction entre le préjudice causé par l'illégalité de la décision de placement dont la réparation relève du juge judiciaire, et le préjudice causé par les fautes commises dans le fonctionnement de l'établissement hospitalier, l'action en responsabilité relevant alors du juge administratif en cas de litige. En 2001, la CAA de Douai ${ }^{36}$ distingue ainsi parmi les préjudices ceux qui résultent d'un internement arbitraire dont la réparation relève de la seule compétence judiciaire et ceux qui en sont détachables car ils résultent de fautes médicales (choix d'un traitement inapproprié) ou de fautes dans l'organisation ou le fonctionnement du service public, telles que l'absence de recherche du consentement au traitement ou le défaut de surveillance du malade ${ }^{37}$.

Mais le Conseil d'État avait retenu, bien avant la réforme de 2011, une formulation très extensive de la compétence judiciaire. Il considérait en effet dans un arrêt du 28 juillet 2000 que le juge judiciaire est compétent pour statuer sur les dommages résultant de «l'ensemble des irrégularités commises à l'occasion ou à la suite de la mesure de placement d'office $»^{38}$, ce qui va donc au-delà des dommages causés par l'illégalité de la décision de placement elle-même et crée une relative incertitude quant au champ exact des dommages à prendre en considération. Doit-on y inclure par exemple les dommages causés par les restrictions illégales à l'exercice de ses droits par le malade? Le Conseil a clairement refusé cette solution en 2007 à propos du préjudice résultant pour le patient des restrictions à son droit de correspondre ${ }^{39}$. Dans ses conclusions, le commissaire du gouvernement $\mathrm{M}^{\mathrm{me}}$ Landais reprend la distinction énoncée plus haut, et s'appuyant sur la décision du Tribunal des conflits rendue le 17 février $1997^{40}$, elle considère:

[...] qu'il appartient au seul juge judiciaire de statuer non pas sur l'ensemble des conséquences dommageables d'une décision d'hospitalisation d'office mais «sur les conséquences dommageables de l'ensemble des irrégularités entachant la mesure de placement d'office ${ }^{41}$.

Or il pourrait en aller différemment aujourd'hui du fait du libellé de l'article L. 3216-1. Celui-ci consacre en effet la compétence judiciaire pour statuer sur la réparation des dommages résultant pour l'intéressé des décisions administratives prises en application des chapitres II à IV, ce qui est plus large que de parler des dommages résultant des irrégularités affectant ces décisions. Combinée à l'idée que la question des droits est étroitement liée à celle de l'admission en soins sans consentement, cette nouvelle formulation plaide là encore pour une extension de la compétence judiciaire en la matière ${ }^{42}$.

En revanche, le juge administratif devrait rester compétent sur les actions en réparation des fautes de surveillance commises par l'hôpital à l'égard d'un patient en soins sans consentement ${ }^{43}$. La jurisprudence antérieure à la réforme montre aussi que le juge administratif peut vérifier à l'occasion de ces actions en réparation la pertinence du traitement médical choisi, en s'appuyant

33. CE, ord., 14 octobre 2004, $\mathrm{n}^{\circ} 273047$ : «la contestation de l'acte par lequel le préfet décide qu'une personne atteinte de troubles mentaux, qui a fait l'objet d'un placement d'office dans un établissement d'hospitalisation spécialisé, doit être transférée dans un autre établissement est soumise à des règles de répartition des compétences entre la juridiction administrative et la juridiction judiciaire, identiques à celles applicables au régime du placement d'office, dans la mesure où le transfert est le corollaire d'un tel placement».

34. Nous retrouverons infra cette question du contrôle du transfert en UMD (I.B).

35. Voir É. Péchillon, «Le pouvoir médical face au refus du consentement...».

36. CAA Douai, 12 juillet 2001, no 98 DAoo 428.

37. Voir par exemple pour des affaires plus récentes: CAA Lyon, 8 avril 2010, nº o LYoo613 (responsabilité de l'hôpital pour le suicide d'une patiente placée en hospitalisation à la demande d'un tiers (HDT) et résultant d'un défaut de surveillance; à cette occasion, le juge vérifie que le traitement médical était adapté); CAA Marseille, 11 juillet 2011, nº 09 MA01562 (responsabilité du CHU de Montpellier pour le décès d'une patiente en placement d'office, résultant d'un défaut dans l'organisation et le fonctionnement de l'établissement).

38. CE, sect., E. A., 28 juillet $2000, \mathrm{n}^{\circ} 151068$ (nous soulignons).

39. CE, 6 avril 2007, n²80494, mentionné aux tables du Recueil Lebon.

40. TC, Préfet de la région Île-de-France, 17 février 1997, nº3045, Recueil Lebon, p. 524.

41. C. Landais, «Les limites du bloc de compétence judiciaire...», conclusions sur CE, 6 avril 2007, n²80494, p. 416: «il appartient au seul juge judiciaire de statuer non pas sur l'ensemble des conséquences dommageables d'une décision d'hospitalisation d'office mais "sur les conséquences dommageables de l'ensemble des irrégularités entachant la mesure de placement d'office”. Le juge judiciaire est donc compétent pour statuer sur les préjudices nés de toute illégalité entachant la décision de placement; or il n'est à aucun moment soutenu dans le présent litige que la décision de placement serait illégale; seule l'illégalité de la décision distincte de restriction du droit de correspondance de l'interné d'office est ici invoquée. Nous pensons donc que les conclusions indemnitaires présentées par M. Bernardet soulèvent un litige distinct de celui qui porterait sur les conséquences dommageables de la décision de placement elle-même».

42. CE, 13 mars $2013, n^{\circ} 354976$, compétence judiciaire pour la réparation du préjudice résultant de l'absence d'information donnée au patient après son admission; CAA Paris, 28 janvier 2013, no 11PA01689.

43. Voir, s'agissant d'une affaire antérieure à la réforme, CAA Marseille, 11 juillet 2011, $\mathrm{n}^{\circ}$ o9MAo1562 pour le décès d'une patiente en placement d'office. 
sur des expertises ${ }^{44}$. De son côté, le juge judiciaire s'est estimé compétent pour statuer sur la réparation des dommages causés par un traitement inapproprié, c'est du moins ce qui ressort d'un arrêt rendu par la cour d'appel de Paris en $2013^{45}$ dans le cadre d'une affaire concernant une décision d'hospitalisation annulée pour illégalité ${ }^{46}$.

La frontière entre ce qui relève de la compétence du juge administratif et ce qui en est exclu est donc particulièrement ténue, elle dépend de la manière dont le juge conçoit ce qui est lié à la décision de placement et ce qui peut s'en détacher. Il existe ainsi plusieurs lectures possibles de l'article L. 3216-1 et de la compétence judiciaire : une lecture restrictive qui laisse au juge administratif le soin de statuer sur tous les litiges relatifs à des agissements ou des décisions autres que celles d'admission et de maintien en soins contraints et une lecture extensive qui inclut dans la compétence judiciaire tout ce qui a trait de près ou de loin à cette admission. Face à ces incertitudes, les deux ordres de juridiction ont parfois tendance à concevoir leur compétence comme complémentaire: lorsque c'est le JLD qui est saisi, il vérifie ce que le juge administratif pourrait être conduit à examiner dans un autre cadre, c'est du moins ce que semble indiquer la jurisprudence relative aux décisions restreignant les libertés des personnes internées. Mais la portée de la protection offerte au patient est d'autant plus incertaine que les juges semblent finalement peu enclins à se charger d'un contentieux délicat et renoncent à statuer sur certaines questions, notamment celles qui sont liées au traitement.

\section{B. Une protection affaiblie par les réticences des juges à statuer sur certaines questions}

Les incertitudes relatives au juge qu'il faut saisir affaiblissent la protection des droits des patients par les complications qu'elles entraînent pour le requérant et parce que le patient risque parfois de ne trouver aucun juge admettant sa compétence pour statuer sur sa demande. Il arrive en outre que le juge compétemment saisi estime que la décision qui lui est déférée n'est pas susceptible de contrôle juridictionnel. Qu'il s'agisse des décisions liées au traitement médical (1), des sorties d'essai et permissions de sortie (2) ou du placement en UMD (3), la protection du patient ne semble pas assurée en l'état de la jurisprudence.

\section{Traitement médical et programme de soins}

Les réticences des juges à statuer sur le traitement médical ou le programme de soins sont particulièrement bien illustrées par l'affaire du Risperdal jugée en 2012 puisque les deux ordres de juridiction se sont prononcés de manière finalement négative quant à leur compétence. Saisi d'un référé-liberté par une patiente qui contestait la décision d'augmenter la dose de son traitement, le juge administratif de première instance a d'abord admis sa compétence et fait droit à sa demande ${ }^{47}$. Mais son ordonnance a été annulée en appel par le Conseil d'État, qui a estimé que:

[...] le juge administratif n'est manifestement pas compétent pour connaître du bien-fondé des décisions prises par les médecins qui participent à la prise en charge de patients faisant l'objet d'une mesure d'admission en soins psychiatriques sans leur consentement ${ }^{48}$.

L'argumentation suivie par le juge des référés du Conseil d'État s'appuyait sur l'idée que le programme de soins est étroitement lié au bien-fondé de la mesure de soins sans consentement dont la contestation relève du seul juge judiciaire. Le Conseil considère en effet que le refus de la contrainte par le patient peut conduire les médecins à le réintégrer dans un régime d'hospitalisation complète, ce qui imposera la saisine systématique du JLD.

44. CAA Douai, 12 juillet 2001, $\mathrm{n}^{\circ}$ 98DAoo428: «si, indépendamment du préjudice né du caractère abusif de son internement, M. Y... a également entendu se prévaloir de préjudices distincts qui résulteraient de fautes médicales ou de fautes dans l'organisation ou le fonctionnement du service public hospitalier révélées par l'aggravation de son stress psychologique, par l'absence de recherche de son consentement au traitement et par l'existence d'un traitement chimiothérapeutique inapproprié, il n'établit pas, en ce qui le concerne, la réalité de ces préjudices".

45. CA Paris, 28 juin 2013, $\mathrm{n}^{\circ}$ 12/O2842 : la responsabilité du centre hospitalier où l'intéressée a été hospitalisée en soins psychiatriques sous contrainte est engagée suite à l'annulation de la décision d'admission qui n'indiquait pas le degré de parenté ou à défaut la nature des relations entre l'intéressée et l'auteur de la demande. La décision d'annulation constitue en effet le fait générateur de l'obligation d'indemniser l'entier préjudice subi par l'intéressée, né de l'atteinte portée à sa liberté par l'hospitalisation irrégulièrement décidée sans qu'il n'y ait lieu de rechercher si la mesure de placement était médicalement justifiée. L'intéressée ne démontre pas avoir reçu un traitement inapproprié ayant comporté des effets dommageables et n'a pas refusé la poursuite de l'hospitalisation après la mainlevée de la mesure prise à son encontre. Le préjudice subi réside donc dans le préjudice moral et le retentissement psychologique lié aux conditions propres à une telle hospitalisation et se trouve indemnisé, en considération de la courte durée du séjour contraint du 12 au 18 août, par l'octroi de 3000 euros de dommages et intérêts.

46. Ce qui signifie ici qu'il s'agissait bien de statuer sur les conséquences dommageables d'une décision d'admission illégale.

47. É. Péchillon, «Le pouvoir médical face au refus de consentement...», note sous TA Rennes, M ${ }^{m e}$ A c. CHS Guillaume Régnier, 18 juin 2012 , $\mathrm{n}^{\circ} 1202373$; voir également C. Castaing, «La volonté... ». Lorsque le juge des référés de Rennes a statué, $\mathrm{M}^{\mathrm{me}} \mathrm{A}$ se trouvait en hospitalisation complète.

48. CE, CHS Guillaume Régnier, 16 juillet 2012, nº 360793, L'actualité juridique. Droit administratif, n 37, 2012, p. 2035; voir également C. Castaing, «La volonté...». Lorsque $\mathrm{M}^{\mathrm{me}} \mathrm{A}$ a fait appel, elle se trouvait désormais en soins ambulatoires sans consentement. Selon le Conseil d’État, "Considérant [...] que le législateur a entendu donner compétence à l'autorité judiciaire pour apprécier le bien-fondé de toutes les mesures de soins psychiatriques sans consentement, qu'elles portent atteinte à la liberté individuelle ou non; que l'appréciation de la nécessité des décisions prises par les médecins qui participent à la prise en charge de personnes qui font l'objet de tels soins, pour autant qu'elle relève du contrôle du juge, de même que, le cas échéant, celle de la capacité de ces personnes à y consentir, sont étroitement liées à celle du bien-fondé des mesures elles-mêmes; qu'en particulier, dans l'hypothèse où un patient qui ne fait pas l'objet d'une hospitalisation complète refuse de se soumettre au traitement jugé nécessaire à son état par l'établissement d'accueil et où la forme de sa prise en charge est par suite modifiée pour procéder à une hospitalisation complète, le juge des libertés et de la détention doit se prononcer sur cette mesure dans un délai de quinze jours; qu'il suit de là que le juge administratif n'est manifestement pas compétent pour connaître du bien-fondé des décisions prises par les médecins qui participent à la prise en charge de patients faisant l'objet d'une mesure d'admission en soins psychiatriques sans leur consentement ». 
En outre le juge judiciaire est compétent pour accorder la mainlevée du régime de soins ambulatoires sans consentement. Il y a donc selon le juge administratif un lien entre la contestation du traitement imposé au patient et celle du régime de soins sans consentement.

Mais le juge judiciaire, saisi à son tour par la patiente quelque temps plus tard, a estimé quant à lui que l'autorité judiciaire n'était pas compétente pour statuer sur le contenu du programme de soins, qu'il s'agisse des doses de médicaments ou du nombre de "permissions de sortie» accordées à la patiente qui se trouvait à l'époque en soins ambulatoires ${ }^{49}$. Au bout du compte, et au vu de ces jurisprudences ${ }^{50}$, seul le JLD est compétent pour statuer sur le principe même d'un programme de soins sans consentement, mais aucun juge ne semble compétent pour statuer sur le contenu de ce programme.

En outre, lors de cette affaire, le juge des référés du Conseil d'État a laissé entendre que quand bien même il ne s'agirait pas de statuer sur le bien-fondé de la mesure de soins sans consentement, la décision en cause ne pourrait pas être contrôlée car «l'appréciation de la nécessité des décisions prises par les médecins", ne relève peut-être pas du contrôle du juge ${ }^{51}$. Comme le souligne Éric Péchillon, il s'agissait d'une invitation faite au juge judiciaire de refuser de contrôler les décisions médicales relatives au traitement ${ }^{52}$. Or il semble que le juge judiciaire ait fait sienne la position du Conseil d'État quant à l'impossibilité de contrôler la décision médicale. Selon la cour d'appel de Bordeaux en effet, les modalités de prise en charge du patient relèvent du strict pouvoir du médecin ${ }^{53}$. De son côté, la cour d'appel de Limoges se contente de contrôler le maintien en hospitalisation d'office et s'abstient de répondre au patient qui conteste les injections d'un médicament affectant sa vie privée ${ }^{54}$.

Pourtant, l'argument selon lequel il s'agit de décisions purement médicales et comme telles insusceptibles de contrôle par quelque juge que ce soit ne convainc pas vraiment: le JLD est en effet conduit à se prononcer sur le bien-fondé de la mesure d'admission en soins sans consentement, ce qui signifie qu'il est conduit à apprécier une décision médicale. S'agissant de malades en pla- cement libre ou internés, le juge administratif accepte depuis longtemps d'examiner si les médecins ont commis des fautes justifiant de mettre en jeu la responsabilité de l'hôpital. Et dans le cadre d'actions en réparation des préjudices causés par une hospitalisation forcée irrégulière, le juge judiciaire est prêt à statuer sur le préjudice résultant d'un traitement inappropriés5. En revanche, au regard des principes qui fondent actuellement la répartition des compétences entre les deux ordres de juridiction, on comprend que le juge judiciaire se soit estimé incompétent pour statuer sur le contenu du programme de soins en ambulatoire dans la mesure où il ne s'agissait ni de statuer directement sur une demande de mainlevée de la décision d'admission en soins sans consentement ni de contrôler une réadmission en hospitalisation complète, ni de réparer un préjudice causé par ces décisions. On peut aussi comprendre que ce type de contestation appelle une expertise toujours coûteuse et problématique dans le cadre d'une action en référé.

Il reste que l'absence de recours permettant de contester le choix du traitement pourrait éventuellement conduire la Cour européenne des droits de l'homme à condamner la France si l'on s'en tient à l'arrêt rendu le 3 juillet 2012 contre la Finlande ${ }^{56}$. Certes, l'affaire portée devant la Cour de Strasbourg concernait l'administration forcée d'un médicament sans garanties légales suffisantes ni recours devant un tribunal. Or, dans le cas d'un programme de soins en ambulatoire, le recours à la contrainte est impossible, sauf à décider la réadmission du patient en hospitalisation complète. Dans le cadre d'une hospitalisation complète en revanche, le choix du traitement imposé et des modalités de son administration peut difficilement être qualifié de mesure d'ordre intérieur contre laquelle le recours n'est pas recevable, il semble pourtant qu'il ne soit contestable que dans le cadre d'actions en réparation des préjudices qu'il aurait pu causer au patient au titre de la faute médicale. Il faut en outre s'interroger sur les limites de cette jurisprudence relative au traitement: que doit-on entendre par décision médicale ou traitement médical? Faut-il penser que le nombre de permissions de sorties octroyées au patient fait partie de ces décisions insusceptibles de contrôle?

49. TGI Rennes, ord. JLD, 14 septembre 2012, nº 12/04081; CA Rennes, ord., 28 septembre 2012, n 12/o0228: «la loi n’a pas donné compétence au juge judiciaire pour apprécier le bien-fondé d'une prescription médicale ou d'une posologie».

50. De même, CA Bordeaux, 3 avril 2013, $1^{\text {er }}$ Président, $n^{\circ}$ 13/01902: "S'il ne relève pas de la compétence du juge judiciaire de déterminer les modalités de prise en charge du patient qui relèvent du strict pouvoir du médecin, force est-il de constater que ce programme de soins est des plus minimalistes en ce qui concerne l'assouplissement du régime d'hospitalisation de Madame P.».

51. CE, ord., 16 juillet 2012, $n^{\circ} 360793$, cons. $7:$ «Considérant qu'il résulte de ce qui précède que le législateur a entendu donner compétence à l'autorité judiciaire pour apprécier le bien-fondé de toutes les mesures de soins psychiatriques sans consentement, qu'elles portent atteinte à la liberté individuelle ou non; que l'appréciation de la nécessité des décisions prises par les médecins qui participent à la prise en charge de personnes qui font l'objet de tels soins, pour autant qu'elle relève du contrôle du juge, de même que, le cas échéant, celle de la capacité de ces personnes à y consentir, sont étroitement liées à celle du bien-fondé des mesures elles-mêmes» (nous soulignons).

52. É. Péchillon, «Légalité d’un dosage médicamenteux: le Conseil d’État renvoie au juge judiciaire le soin de contrôler les conséquences d'une mesure de police», La semaine juridique, édition administrations et collectivités territoriales, $\mathrm{n}^{\circ} 23,3$ juin 2013, chron. $\mathrm{n}^{\circ} 2168$.

53. Voir supra n. 50.

54. CA Limoges, 20 septembre $2013, \mathrm{n}^{\circ} 13 / 00033$.

55. CA Paris, 28 juin 2013, $\mathrm{n}^{\circ} 12 / 02842$ : il faut sans doute admettre que statuer sur le préjudice résultant d'un traitement ne revient pas à statuer sur le choix du traitement; ici le juge statue sur un préjudice résultant du traitement et non sur le choix du traitement lui-même hors préjudice.

56. Cour EDH, X. c. Finlande, 3 juillet 2012, nº 34806/04. 


\section{Sorties d'essai et permissions de sortie}

Lorsque la décision contestée relève de la compétence du juge administratif, encore faut-il donc que le juge ne la qualifie pas de mesure d'ordre intérieur. S'agissant des sorties d'essai, le juge administratif a opéré un revirement de jurisprudence en septembre 2010 en admettant d'examiner le recours contre une autorisation de sortie d'essai ${ }^{57}$ dans un contexte sécuritaire particulièrement marqués8. Le Conseil d'État s'appuyait en l'espèce sur la nature et les effets de l'autorisation de sortie d'essai accordée au malade hospitalisé d'office. Alors que la jurisprudence antérieure les qualifiait de mesures d'ordre intérieur ${ }^{59}$, notamment selon la CAA de Douai parce que « relevant du traitement médical», le juge de 2010, reprenant la jurisprudence applicable aux mesures mises en œuvre dans l'administration pénitentiaire, estime que les autorisations de sortie peuvent être contestées par la voie du recours pour excès de pouvoir (REP) car "par leur nature et par l'importance de leurs effets sur la situation des personnes intéressées et de leur entourage " ${ }^{60}$, elles constituent des décisions faisant grief que les tiers peuvent attaquer, le patient n'ayant quant à lui aucun intérêt à agir contre cette autorisation.

Il n'y a, à première vue, pas de raison pour que les refus de sortie d'essai ne soient pas eux aussi qualifiés de mesures faisant grief: si les autorisations de sortie sont des décisions susceptibles de recours, a fortiori les refus devraient-ils l'être aussi. Néanmoins, la solution est loin d'être certaine. On s'aperçoit en effet que le Conseil d'État fait en 2013 une analyse très restrictive du refus de sortie d'essai dans le cadre d'un litige antérieur à la réforme de 2011: le Conseil semble n'admettre d'examiner le recours que parce que la décision avait pour conséquence en l'espèce de prolonger l'internement sans qu'il y ait eu intervention du juge judiciaire comme l'exige aujourd'hui le législateur pour autoriser ce maintien ${ }^{61}$. Dans des cas différents, il est donc probable que le juge estimerait que le refus n'a pas d'effets suffisants sur la situation du patient pour justifier d'y voir autre chose qu'une mesure d'ordre intérieur.

Mais l'ensemble de cette jurisprudence pourrait en outre se trouver remise en cause si le juge administratif persiste à penser que ces décisions, notamment celles refusant la sortie d'essai, ont trait au bien-fondé de la mesure de soins sans consentement et comme telles relèvent exclusivement de la compétence judiciaire, voire d'aucun juge parce qu'elles relèvent du traitement médical. Sans doute faut-il cependant distinguer selon les situations: $s^{\prime} i$ s'agit d'un programme de soins en ambulatoire dont le Conseil constitutionnel a estimé qu'il ne concerne pas la liberté individuelle au sens de l'article 66, mais seulement la liberté personnelle du patient, le nombre de permissions de sorties fait partie du programme de soins pour un patient qui ne relève plus d'un internement véritable mais d'une hospitalisation partielle. Ici le juge judiciaire ne se prononce pas automatiquement sur la mesure, il n'intervient que s'il est saisi par le requérant ou ses proches contestant la mesure de soins contraints en ambulatoire ou demandant réparation des conséquences dommageables de son maintien. À cette occasion pourrait être contesté le refus de permissions de sorties plus longues ou plus fréquentes, mais aussi les autorisations de sortie lorsque celles-ci ont causé un dommage au patient. La cour d'appel de Rennes dans l'affaire des dosages médicamenteux a cependant exclu la compétence judiciaire pour statuer sur l'organisation des permissions de sortie. Et rien n'assure que le juge administratif accepterait de statuer puisqu'il estime que la question est liée au traitement choisi par les médecins.

\section{Placement en unité pour malades difficiles (UMD)}

Un problème similaire concerne le cas du placement en UMD. Le juge administratif estimait en 2004 que le transfert en UMD est le corollaire du placement d'office et relève par conséquent du contrôle du juge judiciaire

57. CE, 24 septembre 2010, $\mathrm{n}^{\circ}$ 329628, L'actualité juridique. Droit administratif, $\mathrm{n}^{\circ}$ 2, 2011, p. 112 : «par leur nature et l'importance de leurs effets sur la situation des personnes intéressées et de leur entourage, les décisions qui autorisent les sorties d'essai et en fixent les modalités sont susceptibles d'être contestées par la voie du recours pour excès de pouvoir». Le Conseil revient ici sur la jurisprudence issue de CE, 17 novembre 1997, nº 170531 , arrêt dans lequel le Conseil s'estimait compétent mais rejetait le recours car la décision accordant une sortie d'essai constitue selon lui « une mesure relevant du traitement du malade hospitalisé et ne constitue pas une décision susceptible de faire l'objet d'un recours pour excès de pouvoir ».

58. Voir L. Friouret, «De nouvelles perspectives de recours contre les décisions relatives aux sorties d'essai de personnes hospitalisées d'office», commentaire de CE, 24 septembre 2010, $\mathrm{n}^{\circ}$ 329628, L'actualité juridique. Droit administratif, $\mathrm{n}^{\circ}$ 2, 2011, p. 112-116.

59. CE, 17 novembre 1997, $\mathrm{n}^{\circ}$ 170531; CAA Douai, 12 juillet 2001, $\mathrm{n}^{\circ}$ 98DAoo428: ces autorisations sont «des mesures relevant du traitement du malade», elles ne font pas grief.

60. CE, 24 septembre $2010, \mathrm{n}^{\circ} 329628$.

61. CE, 4 octobre 2013, $\mathrm{n}^{\circ} 348858$, cons. 9 et 13: «Considérant que si la décision du directeur de l'hôpital psychiatrique de Naugeat du 6 novembre 1967 a pour objet de mettre fin à la sortie d'essai dont bénéficiait $\mathrm{M}^{\mathrm{me}} \mathrm{A}$... et de prononcer sa réintégration en hospitalisation complète, elle a nécessairement pour effet de prolonger la mesure de placement volontaire prononcée le 12 septembre 1967; que l'adoption de cette décision n'a été rendue possible que par les dispositions des articles L. 337, L. 338, L. 339 et L. 340 du code de la santé publique dans leur rédaction antérieure à la loi du 27 juin 1990, qui permettaient le maintien dans un établissement de soins d'une personne atteinte de troubles mentaux au-delà de quinze jours sans intervention d'une juridiction de l'ordre judiciaire, et que le Conseil constitutionnel a, pour ce motif, déclarées contraires à la Constitution; qu'il résulte de ce qui a été dit précédemment qu'il appartient au Conseil d'État de remettre en cause les effets produits par ces dispositions en relevant que la décision du 6 novembre 1967 est, en conséquence de la décision nº 2011-202 QPC du Conseil constitutionnel, privée de base légale; que, par suite, et sans qu'il soit besoin d'examiner l'autre moyen du pourvoi dirigé contre l'arrêt attaqué en tant qu'il statue sur la légalité de la décision du 6 novembre 1967, $\mathrm{M}^{\mathrm{me}}$ A... est fondée à en demander, dans cette mesure, l'annulation; [...] la décision attaquée, compte tenu de ses effets décrits ci-dessus, est susceptible d'être contestée par la voie du recours pour excès de pouvoir» (nous soulignons). 
en cas de recours du patient ${ }^{62}$. Or la jurisprudence judiciaire laisse penser que le JLD refuse le plus souvent de contrôler la décision du préfet de transférer un patient en UMD alors même que celui-ci la conteste ${ }^{63}$. Cette situation est évidemment peu propice à la protection des droits du malade. Il existe potentiellement ici un vide juridique défavorable au patient dont le transfert en UMD pourrait s'avérer injustifié s'il n'est pas dangereux pour les autres malades ni pour les soignants ou si ce transfert entraîne des conséquences disproportionnées pour le patient et ses proches ${ }^{64}$. Faut-il en conclure que le juge judiciaire renvoie au juge administratif le soin de statuer sur la légalité du placement en UMD notamment au regard du droit à la vie familiale de l'intéressé, ou de la nécessité de cette contrainte supplémentaire? Dans sa décision QPC rendue le 14 février 2014 sur saisine de la Cour de cassation, le Conseil constitutionnel nie, de manière contestable, toute spécificité au placement en UMD puisqu'il estime que:

[...] le régime juridique de privation de liberté auquel sont soumises les personnes prises en charge dans une unité pour malades difficiles n'est pas différent de celui applicable aux autres personnes faisant l'objet de soins sans leur consentement sous la forme d'une hospitalisation complète $[\ldots]^{65}$.

Il s'ensuit que le patient peut certes demander à tout moment au JLD la mainlevée de la mesure de placement, mais que rien n'impose au juge de statuer spécifiquement sur le placement en UMD. Le Conseil constitutionnel comme le législateur de 2013 voit donc dans le placement en UMD une simple mesure interne à l'organisation du service de soins psychiatriques ${ }^{66}$. Une protection complémentaire peut dès lors être offerte au patient par le juge administratif; celui-ci accepte en effet depuis 1994 de voir dans les transferts d'établissement des décisions faisant grief lorsqu'ils ont des effets sur la vie familiale du patient et de ses proches ${ }^{67}$. A fortiori devrait-il en aller de même du placement en UMD, le juge pouvant alors statuer sur la nécessité du transfert en UMD au regard de la dangerosité du patient.

Quoiqu'il puisse parfois conduire à une certaine complémentarité de la protection offerte par chacun des ordres de juridiction, le système de répartition actuel s'avère donc à la fois d'une extrême complexité et d'une grande incertitude, ce qui n'est guère satisfaisant au regard de l'exigence de recours effectif. Il n'est par conséquent pas toujours protecteur des droits des individus, contrairement aux intentions affichées par l'article 66 de la Constitution. Les problèmes posés par la dualité des ordres de juridiction susceptibles d'intervenir justifient donc à notre sens de repenser les fondements de ce système de répartition.

\section{Un système de protection à repenser?}

Les difficultés pour le malade et ses proches de trouver un juge susceptible de statuer sur les décisions qui le concernent subsistent malgré la réforme. Dès lors, elles conduisent à s'interroger sur la possibilité de penser autrement la répartition des compétences de façon à protéger au mieux les droits des malades (B), ce qui nécessite au préalable de remettre en perspective les fondements de la répartition actuelle $(\mathrm{A})$.

\section{A. Les fondements contestables de la répartition actuelle}

Pour déterminer la juridiction compétente en cas de restriction à la liberté des administrés par une décision administrative, le législateur et les juges doivent combiner plusieurs principes potentiellement contradictoires mais qui sont tous de rang constitutionnel. Or, les résultats de cette conciliation apparaissent contestables en ce qu'ils ne permettent pas de garantir efficacement les droits des individus (1). En outre, certains de ces principes reposent sur des présupposés devenus obsolètes (2).

62. CE, ord., 14 octobre $2004, \mathrm{n}^{\circ} 273047$ : «la contestation de l'acte par lequel le préfet décide qu'une personne atteinte de troubles mentaux, qui a fait l'objet d'un placement d'office dans un établissement d'hospitalisation spécialisé, doit être transférée dans un autre établissement est soumise à des règles de répartition des compétences entre la juridiction administrative et la juridiction judiciaire, identiques à celles applicables au régime du placement d'office, dans la mesure où le transfert est le corollaire d'un tel placement ».

63. Voir TGI de Brive-la-Gaillarde, 29 mai 2013: «il ne relève pas de la compétence du JLD d’orienter un patient vers une structure de soins», cité par CA Limoges, 17 juin 2013, nº 13/ooo25; CA Limoges, 8 octobre 2013, n 13/00036: ici le JLD confirme l'hospitalisation complète du patient sans statuer sur sa contestation du placement en UMD.

64. Voir CA Limoges, 8 octobre 2013, no 13/00036: dans le cadre d'un contrôle systématique, le JLD confirme le maintien de l'hospitalisation complète sans se prononcer sur le placement en UMD pourtant contesté par le patient alors même que l'hôpital reconnaît son absence d'agressivité et que ce placement l'éloigne de sa famille. Voir également CA Limoges, 17 juin 2013, n 13/00025.

65. CC, déc. $n^{\circ}$ 2013-367 QPC du 14 février 2014, Consorts L., cons. 10. Dès lors le Conseil estime, sans vraiment convaincre, qu’il n'y a pas d'incompétence négative du législateur lorsque ce dernier renvoie au décret le soin de fixer les modalités de prise en charge des patients en UMD, ce qui peut sembler pourtant peu compatible avec la décision 2012-235 QPC du 20 avril 2012, Association Cercle de réflexion et de proposition d'actions sur la psychiatrie. La loi du 27 septembre 2013 ayant supprimé toute référence aux UMD, le régime de ces unités relève donc aujourd'hui uniquement du pouvoir réglementaire (voir le décret n 2011-847 du 18 juillet 2011 et l'arrêt CE, CRPA, 20 décembre 2013, n 352668).

66. Voir notamment J. Le Menn, Rapport fait au nom de la commission des affaires sociales sur la proposition de loi, adoptée par l'Assemblée nationale après engagement de la procédure accélérée, visant à modifier certaines dispositions issues de la loi no $2011-803$ du 5 juillet 2011 relative aux droits et à la protection des personnes faisant l'objet de soins psychiatriques et aux modalités de leur prise en charge, n 835 , enregistré à la présidence du Sénat le 11 septembre 2013, session extraordinaire 2012-2013, p. 9-10.

67. Voir CE, 25 mai 1994, n 143702 et 143949. Il l'a admis également lorsque ce transfert a pour but de placer le malade dans un établissement pourvu d'une UMD (CE, sect., E. A., 28 juillet $\left.2000, \mathrm{n}^{\circ} 151068\right)$. 


\section{La combinaison problématique de principes contradictoires}

La séparation des autorités administratives et judiciaires ${ }^{68}$ sur laquelle repose l'existence même de la juridiction administrative a indirectement donné naissance à un principe fondamental reconnu par les lois de la République consacré par le Conseil constitutionnel en 1987: celui de la compétence réservée du juge administratif pour annuler ou réformer des décisions prises par des personnes publiques dans l'exercice de prérogatives de puissance publique ${ }^{69}$. Les décisions d'admission et de maintien en soins sans consentement appartiennent précisément à cette catégorie de décision. Mais le Conseil a pris soin d'énoncer deux exceptions à la réserve de compétence du juge administratif: les matières réservées par nature à l'autorité judiciaire d'une part, les exigences d'une bonne administration de la justice d'autre part $^{7^{\circ}}$. Dans ces deux hypothèses, le juge judiciaire peut, voire doit $^{77}$, être reconnu compétent pour statuer sur les litiges qui relèveraient normalement du juge administratif. Aucune liste officielle n'a jamais été donnée pour savoir précisément quelles sont les matières réservées par nature à l'autorité judiciaire, mais la tradition et surtout l'article 66 de la Constitution conduisent à penser que la protection de la liberté individuelle en fait partie. Cette liberté individuelle est aujourd'hui conçue de manière restrictive: il s'agit essentiellement, voire uniquement de la protection contre les détentions arbitraires, autrement dit de la protection de la sûreté ${ }^{72}$. Or les décisions relatives à l'hospitalisation forcée d'un patient relèvent clairement de ce cas de figure.

L'article 66 de la Constitution se contente d'imposer au législateur de prévoir l'intervention obligatoire de l'autorité judiciaire (plus précisément ici celle du juge judiciaire) pour autoriser la prolongation d'une restriction à la sûreté; il ne concerne pas directement la répartition des compétences entre les deux ordres en cas de litige. Mais cette intervention obligatoire qui se traduit par l'examen systématique par le JLD des décisions d'hospitalisation complète sans consentement entraîne presque nécessairement la compétence du juge judiciaire en cas de litige sur le même sujet: il serait paradoxal de lui refuser la compétence pour statuer sur la demande de mainlevée de la mesure de soins sans consentement ${ }^{73}$ alors qu'il est obligé de statuer sur l'hospitalisation complète dans les douze jours du prononcé de la mesure, puis dans les six mois suivant la décision de maintien ${ }^{74}$. L'argument de la bonne administration de la justice s'ajoute ici à l'article 66 pour fonder cette attribution de compétence. On devrait même considérer que le juge judiciaire a également le droit d'annuler lui-même la décision administrative privative de liberté 75 puisque les «matières réservées par nature à l'autorité judiciaire» justifient d'échapper à la compétence réservée au juge administratif d'annuler les décisions de la puissance publique.

S'agissant des recours contre les décisions relatives à l'admission en soins ambulatoires contraints, il n'y a guère aujourd'hui que l'argument de la bonne administration de la justice qui justifie la compétence du juge judiciaire

68. Principe résultant de la loi des 16 et 24 août 1790 interdisant aux tribunaux judiciaires de s'immiscer dans les opérations des corps administratifs.

69. CC, déc. no 86-224 DC du 23 janvier 1987, Loi transférant à la juridiction judiciaire le contentieux des décisions du Conseil de la concurrence, cons. 15: «conformément à la conception française de la séparation des pouvoirs, figure au nombre des "principes fondamentaux reconnus par les lois de la République" celui selon lequel, à l'exception des matières réservées par nature à l'autorité judiciaire, relève en dernier ressort de la compétence de la juridiction administrative l'annulation ou la réformation des décisions prises, dans l'exercice des prérogatives de puissance publique, par les autorités exerçant le pouvoir exécutif, leurs agents, les collectivités territoriales de la République ou les organismes publics placés sous leur autorité ou leur contrôle». Voir L'actualité juridique. Droit administratif, nº 5, 1987, p. 346, note J. Chevallier; Revue française de droit administratif, 1987, p. 287, note B. Genevois et p. 301, note L. Favoreu; Revue du droit public, 1987, p. 1341, note Y. Gaudemet; Dalloz, 1988 , p. 117, note F. Luchaire.

70. Ibid., cons. 16: «Considérant cependant que, dans la mise en œuvre de ce principe, lorsque l'application d'une législation ou d'une réglementation spécifique pourrait engendrer des contestations contentieuses diverses qui se répartiraient, selon les règles habituelles de compétence, entre la juridiction administrative et la juridiction judiciaire, il est loisible au législateur, dans l'intérêt d'une bonne administration de la justice, d'unifier les règles de compétence juridictionnelle au sein de l'ordre juridictionnel principalement intéressé».

71. C'est du moins ce qu'impliquerait logiquement la formulation du considérant 15 précité pour l'exception des matières réservées par nature à l'autorité judiciaire; sur ce point, voir notamment L'actualité juridique. Droit administratif, nº 5, 1987, p. 345, note J. Chevallier. S'agissant en revanche des exigences de bonne administration de la justice, le Conseil autorise le législateur à donner compétence au juge judiciaire au lieu de respecter la compétence réservée du juge administratif, mais en 1987 il s'agit d'une simple faculté ouverte au législateur. On pourrait y voir aujourd'hui une obligation au titre du droit à un recours effectif et parce que la bonne administration de la justice est désormais un objectif constitutionnel (CC, déc. n² 2009-595 DC du 3 décembre 2009, Loi organique relative à l'application de l'article 61-1 de la Constitution).

72. Voir l'évolution de la jurisprudence constitutionnelle, notamment CC, déc. nº 2003-467 du 13 mars 2003, Loi pour la sécurité intérieure, cons. 8. Voir également G. Armand, L'autorité judiciaire gardienne de la liberté individuelle dans la jurisprudence du Conseil constitutionnel, thèse de doctorat, université de Caen Basse-Normandie, 2000 (dactyl.).

73. Voir art. L. 3211-12 CSP.

74. Voir art. L. 3211-12-1 CSP dans sa version issue de la loi du 27 septembre 2013.

75. Mais le juge judiciaire semble ne pas s'y sentir autorisé, car l'article L. 3216-1 CSP ne lui donne pas explicitement ce pouvoir. Voir notamment M. Vialettes, M. Grosset, «L'unification du contentieux de l'hospitalisation sans consentement... », n 14 : «le juge des libertés et de la détention prononce la mainlevée de la mesure, l'article L. 3216-1 ne lui confiant pas d'autre pouvoir, et notamment pas celui, par exemple, d'enjoindre à l'administration ou à l'établissement hospitalier de régulariser la procédure, ou encore, d'annuler ou de réformer la décision litigieuse». Voir également A. Pena, «Internement psychiatrique... », p. 966: «au vu du principe de séparation des autorités administratives et judiciaires, [la compétence judiciaire] doit permettre au juge judiciaire d'écarter la décision administrative qu'il estime illégale mais en aucun cas ne l'autorise à l'annuler ou à la réformer, le but étant de remettre en liberté une personne qui n'a légalement aucune raison d'être enfermée». 
pour statuer sur la légalité de ces décisions et sur la réparation de leurs conséquences dommageables. Le Conseil constitutionnel $^{76}$, puis le législateur ${ }^{77}$ ont en effet relevé que les soins ne pourraient pas être administrés sous la contrainte sans réintégrer la personne en hospitalisation complète, ces décisions ne concernent donc pas directement la sûreté, mais seulement la liberté personnelle du patient qui n'est pas visée par l'article 66.

De même, c'est au titre de la bonne administration de la justice que le législateur a choisi de confier au seul juge judiciaire le soin de statuer sur tous les aspects de l'action en réparation des dommages causés par la décision illégale ${ }^{78}$. La nouvelle compétence attribuée au juge judiciaire pour statuer sur la légalité externe des décisions d'admission et de maintien lui a d'ailleurs donné l'occasion de se réapproprier la jurisprudence administrative en la matière ${ }^{79}$, jurisprudence qui s'était révélée de plus en plus protectrice au cours de ces dernières années ${ }^{80}$.

Mais dès que l'on sort de l'intervention judiciaire imposée par l'article 66 et de l'argument d'une bonne administration de la justice, seul le principe de la compétence réservée du juge administratif est applicable. C'est à la fois le principe de séparation des autorités et l'argument de la compétence technique du juge administratif pour manier les règles du droit administratif qui justifient cette compétence réservée qualifiée par le Conseil constitutionnel de principe fondamental reconnu par les lois de la République. Or, nous l'avons vu, toute la question est de savoir où s'arrête la notion de décisions prises au titre de l'admission en soins sans consentement.
Ce sont des principes identiques qui gouvernent la répartition des compétences juridictionnelles en droit des étrangers ${ }^{81}$, mais avec des résultats assez différents: en droit des étrangers, l'article 66 impose l'intervention du juge judiciaire pour autoriser la prolongation des décisions administratives de rétention ${ }^{82}$ ou de maintien en zone d'attente ${ }^{83}$, mais le juge judiciaire ne peut pas contrôler à cette occasion la légalité de ces décisions administratives. Il doit se contenter de vérifier la régularité de sa saisine et le respect de certains droits de l'étranger sans même pouvoir examiner la légalité ni de la décision de rétention ${ }^{84}$, ni des décisions d'éloignement ou de refus d'entrée qui la fondent ${ }^{85}$. Le recours contre la décision initiale de rétention ou contre la décision de maintien de l'étranger en zone d'attente relève de la seule compétence du juge administratif. Pourtant, comme le rappelle Annabelle Pena:

[...] pour décider de la remise en liberté immédiate de la personne arbitrairement détenue, le juge judiciaire doit pouvoir s'appuyer sur l'illégalité de l'acte administratif à l'origine de la privation de liberté qu'il a lui-même constatée, pour la simple et bonne raison que la plénitude de compétence devient une condition inhérente à l'exercice d'un contrôle effectif et complet du juge judiciaire [...]. La protection de la liberté individuelle se fond ici d'une certaine manière dans la garantie du droit à un recours effectif ${ }^{86}$.

La réforme de 2011 relative aux soins sans consentement permet au juge judiciaire d'effectuer lui-même pleinement ce contrôle, contrairement à ce qui se pro-

76. Voir CC, déc. n 2012-235 QPC du 20 avril 2012, cons. 12: «en permettant que des personnes qui ne sont pas prises en charge en "hospitalisation complète" soient soumises à une obligation de soins psychiatriques pouvant comporter, le cas échéant, des séjours en établissement, les dispositions de l'article L. 3211-2-1 n'autorisent pas l'exécution d'une telle obligation sous la contrainte; que ces personnes ne sauraient se voir administrer des soins de manière coercitive ni être conduites ou maintenues de force pour accomplir les séjours en établissement prévus par le programme de soins; qu'aucune mesure de contrainte à l'égard d'une personne prise en charge dans les conditions prévues par le $2^{\circ}$ de l'article L. $3211-2-1$ ne peut être mise en œuvre sans que la prise en charge ait été préalablement transformée en hospitalisation complète; que, dans ces conditions, le grief tiré de la violation de la liberté individuelle manque en fait».

77. Loi no 2013-869 du 27 septembre 2013, art. 1 et art. L. 3211-12-1, III CSP.

78. Voir art. L. 3216-1 CSP. Cependant, l’appréciation de la légalité d'une décision n'équivaut pas à son annulation, or la compétence réservée du juge administratif ne concerne que l'annulation, elle ne s'étend pas théoriquement à l'appréciation de légalité, même s'il est vrai qu'en matière de décision individuelle, la mise à l'écart de la décision pour illégalité équivaut presque en pratique à son annulation.

79. CA Douai, ord., $M^{m e} X, D, 7$ mars 2013, $\mathrm{n}^{\circ}$ 13/ooo14, Gazette du palais, no 311, 7 novembre 2013, p. 10, note Y. Benhamou.

80. Voir CE, 9 novembre 2001, n 235247 sur le contenu de la motivation obligatoire et CE, 27 mai $2011, n^{\circ} 330267$ sur l'applicabilité de l'article 24 de la loi du 12 avril 2000 relatif à la procédure contradictoire. Voir également M. Vialettes, M. Grosset, "L'unification du contentieux de l'hospitalisation sans consentement...».

81. Voir CC, déc. no 89-261 DC du 28 juillet 1989, Loi relative aux conditions de séjour et d'entrée des étrangers en France, L'actualité juridique. Droit administratif, $\mathrm{n}^{\circ}$ 10, 1989, p. 619-627, note J. Chevallier; Revue française de droit administratif, 1989, p. 691, note B. Genevois; Gazette du palais, 25-27 février 1990, p. 2, note F. Osman; Dalloz, 1990, Jur., p. 161, note X. Prétot.

82. Voir les art. L. 552-1 sq. du Code de l'entrée et du séjour des étrangers et du droit d'asile (CESEDA).

83. Voir les art. L. 222-1 sq. CESEDA.

84. Voir notamment Cass., $2^{\mathrm{e}}$ civ., Préfet de Seine-Saint-Denis, 20 avril 1989, Bulletin civil II, nº 92, p. 45.

85. CC, déc. no 89-261 DC du 28 juillet 1989: «25. Considérant toutefois, que la compétence ainsi reconnue à l'autorité judiciaire pour contrôler une mesure de surveillance qui met en cause la liberté individuelle, s'exerce indépendamment du contrôle de la légalité des décisions administratives de refus d'accès au territoire national, de reconduite à la frontière ou d'expulsion; qu'au demeurant, une mesure de rétention de l'étranger qui est dans l'impossibilité de déférer immédiatement à une décision d'éloignement ne peut intervenir que "s'il y a nécessité absolue"; que dès lors, la prolongation par l'autorité judiciaire de cette mesure de surveillance ne saurait revêtir un caractère systématique et s'appliquer, tant s'en faut, à tous les cas où il y a intervention d'une décision administrative d'éloignement d'un étranger du territoire national; 26 . Considérant que si l'entrée et le séjour irréguliers en France d'un étranger constituent, dans les cas visés aux articles 19 et 27 de l'ordonnance du 2 novembre 1945 modifiée, une infraction pénale relevant de la seule compétence du juge judiciaire, cette compétence spécifique ne saurait justifier qu’il soit fait échec à la compétence générale du juge administratif dans le domaine de l'annulation des actes de la puissance publique».

86. A. Pena, «Internement psychiatrique... », p. 965. 
duit en droit des étrangers ${ }^{87}$. Les différences entre cette répartition et celle qui concerne les patients en soins sans consentement tiennent en grande partie à l'ancienneté des textes relatifs à l'internement des malades mentaux: nous l'avons vu, la compétence du juge judiciaire pour statuer sur le bien-fondé (donc la légalité interne) de la décision d'internement remonte à la loi Esquirol, alors que les recours en matière de police des étrangers ont toujours relevé de la compétence du juge administratif; ce n'est qu'en 1980 que le Conseil constitutionnel a exigé du législateur qu'il prenne en compte l'article 66 en cas de restriction à la sûreté des étrangers ${ }^{88}$ et le législateur s'est alors contenté de prévoir l'intervention de l'autorité judiciaire pour autoriser la prolongation de ces restrictions sans s'intéresser aux recours susceptibles d'être exercés pour en contester la légalité.

En 1989 cependant, le législateur a souhaité confier au juge judiciaire le soin de statuer sur la légalité des arrêtés de reconduite à la frontière qui constituaient le fondement de la plupart des décisions de rétention des étrangers dans l'attente de mettre à exécution la décision d'éloignement ${ }^{89}$. Mais le Conseil constitutionnel a refusé cette solution au motif d'une part que la mesure d'éloignement ne restreint pas la sûreté de l'étranger, mais seulement sa liberté d'aller et venir dont la protection ne relève pas de l'article $66^{90}$; d'autre part que la bonne administration de la justice ne justifiait pas ici de confier ce contentieux au juge judiciaire $^{91}$. La solution ainsi retenue par le Conseil en 1989 a conduit le juge judiciaire à restreindre excessivement sa compétence dans le contrôle qu'il exerce sur la rétention et le maintien en zone d'attente, mettant ainsi en doute l'utilité de l'article 66 comme moyen efficace de protection de la sûreté de l'étranger ${ }^{92}$.

Le système de répartition des compétences entre les deux ordres de juridiction n'est donc pas très protecteur des droits des individus, il n'est pas non plus très cohérent puisqu'il ne conduit pas du tout à la même répartition pour les malades mentaux et pour les étrangers, malgré des atteintes assez proches à la sûreté. Il est d'autant moins cohérent que le juge judiciaire ne s'estime pas compétent pour annuler une décision administrative échappant pourtant, du fait des exceptions prévues par le juge constitutionnel, à la compétence réservée.

S’agissant des soins sans consentement, la cohérence est encore affaiblie par l'intrusion du juge judiciaire dans des décisions relevant non plus de la liberté individuelle mais de la liberté personnelle ${ }^{93}$, exclue pourtant du champ de l'article 66. Cette situation résulte du hiatus entre l'intention initiale du législateur de 2011 et la jurisprudence constitutionnelle. Lorsque le législateur a conçu les soins ambulatoires sans consentement, il s'agissait de contraindre le patient à des soins, ce qui, vraisemblablement, pouvait déboucher sur une intrusion à son domicile ou sur une hospitalisation partielle forcée. Une telle conception des choses justifiait que le juge judiciaire soit compétent pour statuer sur une demande de mainlevée de la mesure de soins ambulatoires contraints, mais elle aurait dû impliquer plus encore l'intervention systématique du JLD pour autoriser la mise en place de ce programme de soins. Or le législateur de 2011 s'est contenté d'imposer le contrôle systématique du JLD uniquement en cas d'hospitalisation complète du patient, ce qui ne semblait pas conforme aux exigences de l'article 66. C'est l'une des raisons pour lesquelles une QPC a été posée devant le Conseil d'État qui l'a transmise au Conseil constitutionnel. Dans sa réponse, le Conseil a clairement exclu tout recours à la contrainte pour l'administration de soins en ambulatoire ${ }^{94}$; dès lors, la liberté individuelle du patient n'était plus susceptible d'être mise en péril directement par le programme de soins en ambulatoire, seule sa liberté personnelle était concernée. Le contrôle systématique du JLD sur l'admission en soins ambulatoires en amont de tout litige n'est donc pas nécessaire. Néanmoins, le refus du patient de se soumettre aux soins prescrits peut

87. Cependant la loi 2011-672 dite loi Besson du 16 mai 2011 (art. 48 et 52) a inversé les priorités d'intervention entre juge administratif et juge judiciaire, ce qui permet désormais à l'étranger placé en rétention de faire vérifier la légalité de cette décision et de celles qui la fondent par le juge administratif avant que le juge judiciaire ne soit éventuellement saisi par l'administration d'une demande de prolongation de la rétention (voir les art. L. 512-1 III et L. 552-1 CESEDA).

88. CC, déc. n 79-109 DC du 9 janvier 1980, Loi relative à la prévention de l'immigration clandestine.

89. Loi relative aux conditions de séjour et d'entrée des étrangers en France adoptée en dernière lecture par l'Assemblée nationale le 4 juillet 1989 dont l'article 10 insérait dans le texte de l'ordonnance nº 45-2658 du 2 novembre 1945 un article 22 bis ainsi rédigé: «L'arrêté de reconduite peut être contesté par l'étranger qui en fait l'objet devant le président du tribunal de grande instance ou son délégué, qui est saisi sans forme dans les vingt-quatre heures suivant la notification de l'arrêté préfectoral de reconduite et statue selon les formes applicables au référé dans un délai de quarante-huit heures $[\ldots] »$.

90. Motif implicite dans CC, déc. no 89-261 DC du 28 juillet 1989, cons. 24 et 25.

91. CC, déc. 89-261 DC du 28 juillet 1989, cons. 29: "Considérant par ailleurs, que la bonne administration de la justice commande que l’exercice d'une voie de recours appropriée assure la garantie effective des droits des intéressés; que, toutefois, cette exigence, qui peut être satisfaite aussi bien par la juridiction judiciaire que par la juridiction administrative, ne saurait à elle seule autoriser qu'il soit porté atteinte à un principe de valeur constitutionnelle».

92. Sur cette question, voir la thèse de C. Pouly, Droit à la sûreté et police des étrangers. Contribution à l'étude des garanties de la liberté individuelle, thèse de doctorat, université Paris 11, 2003 (dactyl.).

93. La notion de liberté personnelle, explicitement utilisée par le Conseil constitutionnel en 1988 (déc. n 88-244 DC du 20 juillet 1988, Loi portant amnistie), et surtout à partir de 2003 (déc. no $2003-484$ DC du 20 novembre 2003 , Loi relative à la maîtrise de l'immigration, au séjour des étrangers en France et à la nationalité), recouvre la liberté d'aller et venir, le respect de la vie privée, la liberté du mariage. Elle est fondée sur les articles 2 et 4 de la Déclaration des droits de l'homme et du citoyen (déc. n² 2003-467 DC du 13 mars 2003 et 2003-484 DC du 20 novembre 2003 ).

94. CC, déc. $\mathrm{n}^{\circ}$ 2012-235 QPC du 20 avril 2012, Droit administratif, $\mathrm{n}^{\circ} 6$, juin 2012, p. 36-41, note C. Castaing; É. Péchillon, «Censure partielle de la loi du 5 juillet 2011 relative aux soins sous contrainte: vers une réforme en profondeur de la psychiatrie avant octobre 2013? ", La semaine juridique, édition administrations et collectivités territoriales, $\mathrm{n}^{\circ} 26,2$ juillet 2012, chron. $\mathrm{n}^{\circ} 2230$. 
conduire à sa réintégration en hospitalisation complète, créant ainsi un lien entre le soin ambulatoire sans consentement et la liberté individuelle du patient. Le maintien de la compétence judiciaire en aval pour statuer notamment sur les demandes de mainlevée du programme de soins ambulatoires peut dès lors se justifier par les exigences d'une bonne administration de la justice. Mais si l'on tient vraiment à cette bonne administration de la justice, la logique impliquerait d'aller bien au-delà de ce qu'a prévu le législateur et de confier la totalité du contentieux relatif aux soins sans consentement à un seul et même juge, le juge judiciaire.

\section{Des fondements dépassés}

Quoique l'article 66 de la Constitution ne concerne pas directement la compétence juridictionnelle en cas de recours contre la décision administrative restreignant la sûreté de l'individu, il a inévitablement un impact essentiel sur la répartition des compétences entre les deux ordres; et si l'on estime que celle-ci pose problème au regard des droits des administrés, il convient de réfléchir à la question de la pertinence de cette disposition constitutionnelle. En d'autres termes, il faut tenter de comprendre les raisons pour lesquelles le constituant a confié à l'autorité judiciaire la protection de la liberté individuelle.

Introduisant une sorte d'habeas corpus dans la constitution de $1958^{95}$, l'article 66 reprend à son compte la tradition héritée du XIX ${ }^{\mathrm{e}}$ siècle selon laquelle le juge judiciaire est mieux à même que le juge administratif de protéger les libertés des administrés contre l'arbitraire de l'administration. Or cette tradition repose sur deux arguments aujourd'hui largement dépassés: celui de la dépendance du juge administratif vis-à-vis de l'administration active ${ }^{96}$, celui de la moindre efficacité de son intervention.

Si l'on comprend bien la défiance qu'a longtemps pu inspirer le juge administratif aux administrés, celle-ci est juridiquement plus difficile à mettre en avant aujourd'hui dans la mesure où son indépendance, comme celle des juridictions judiciaires, est consacrée au niveau constitutionnel ${ }^{97}$. La jurisprudence antérieure à la réforme montre au surplus que le juge administratif a su protéger les droits des patients: qu'il s'agisse de l'évolution jurisprudentielle en matière de motivation des décisions d'internement ${ }^{98}$ ou de respect du contradictoire ${ }^{99}$, voire de l'admission des recours contre ce qui était quelques années plus tôt considéré encore comme mesures d'ordre intérieur (les sorties d'essai ${ }^{100}$, les transferts d'établissement), l'évolution de la jurisprudence permet de penser que le juge administratif n'est pas moins protecteur des libertés que ne l'est le juge judiciaire. Sans doute faut-il nuancer cette affirmation : l'on sait que les associations de défense des droits des étrangers se sont émues du dispositif mis en place par la loi Besson en $2011^{101}$, dispositif qui a pu être analysé comme un moyen d'éviter la saisine du juge judiciaire ${ }^{102}$; on sait aussi que la jurisprudence administrative est souvent critiquée pour sa sévérité à l'égard des étrangers. Mais pour opérer valablement la comparaison entre les deux juridictions, il faudrait que chacun des juges ait eu l'occasion de se prononcer exactement sur le même litige, ce qui n'est pas le cas en ce domaine, contrairement à celui des soins psychiatriques.

C'est surtout l'argument de la moindre efficacité du recours au juge administratif qui semble aujourd'hui obsolète. La procédure contentieuse a en effet été l'objet de réformes successives depuis la fin du $\mathrm{XX}^{\mathrm{e}}$ siècle qui donnent au juge administratif les moyens d'agir rapidement et d'adresser des injonctions à l'administration ${ }^{103}$. La réforme des procédures de référé et l'introduction du référé-liberté par la loi du 30 juin $2000^{104}$ constituent de ce point de vue une véritable révolution; en effet, le juge administratif des référés peut désormais, dans les quarante-huit heures de sa saisine, enjoindre à l'administration de prendre toute mesure nécessaire à la sauvegarde d'une liberté fondamentale à laquelle elle aurait porté une atteinte grave et manifestement illégale ${ }^{105}$. Dans ce cadre, le juge administratif pourrait protéger la liberté individuelle

95. Voir «l'amendement Waline», notamment, in Documents pour servir à l’histoire de l'élaboration de la Constitution du 4 octobre 1958, vol. II, Paris, La documentation française, 1988, p. 447 sq.; et les discussions: ibid., vol. III, Paris, La documentation française, 1991, p. 167-169 et p. $383-388$. Il s'agissait alors essentiellement de donner une coloration libérale à la Constitution dans un contexte autoritaire, sans pour autant empêcher toute possibilité d'internement administratif.

96. Voir J. Rivero, «Dualité de juridictions et protection des libertés», Revue française de droit administratif, 1990, p. 734: «œuvre d'un pouvoir autoritaire, [la juridiction administrative] ne pouvait [aux yeux des libéraux] que le servir, et il eût été illusoire d'attendre d'elle qu'elle puisse assurer contre lui la protection des libertés» (cité par A. Farinetti, «L’unification du contentieux...», p. 111).

97. CC, déc. $\mathrm{n}^{\circ}$ 80-119 DC du 22 juillet 1980, Loi portant validation d'actes administratifs, cons. 6: «Considérant qu'il résulte des dispositions de l'article 64 de la Constitution en ce qui concerne l'autorité judiciaire et des principes fondamentaux reconnus par les lois de la République en ce qui concerne, depuis la loi du 24 mai 1872, la juridiction administrative, que l'indépendance des juridictions est garantie ainsi que le caractère spécifique de leurs fonctions sur lesquelles ne peuvent empiéter ni le législateur ni le Gouvernement».

98. Voir CE, 9 novembre 2001, $\mathrm{n}^{\circ} 235247$, Les petites affiches, $\mathrm{n}^{\circ} 67,3$ avril 2002, p. 13, concl. S. Boissard.

99. CE, 27 mai 2011, n 330267 .

100. CE, 24 septembre 2010, $\mathrm{n}^{\circ} 329628$ pour des autorisations de sortie d'essai. Contra: CE, CHS d'Erstein, 17 novembre 1997, nº 170531 . Voir également $\mathrm{CE}, 4$ octobre $2013, \mathrm{n}^{\circ} 348858$, pour la décision de mettre fin à une sortie d'essai.

101. Voir supra n. 87.

102. Voir notamment «Pourquoi il faut combattre le projet de la loi Besson», juillet 2010, http://www.gisti.org/IMG/pdf/hc_ucij_mini-analyse_2010-o7.pdf, p. 9.

103. Voir notamment la loi nº 95-125 du 8 février 1995 relative à l'organisation des juridictions et à la procédure civile, pénale et administrative. 104. Loi $\mathrm{n}^{\circ}$ 2000-597 relative au référé devant les juridictions administratives.

105. Art. L. 521-2 du Code de justice administrative (CJA). 
de manière tout aussi efficace que le juge judiciaire ${ }^{106}$. C'est sans doute ce raisonnement qu'avaient tenté de mener les requérants à partir de 2001 pour contester certaines mesures relatives aux malades internés, mais ils se sont heurtés à la réserve de compétence du juge judiciaire en la matière ${ }^{107}$.

Il n'est pas exclu que les juges veuillent penser leur compétence sinon comme concurrente, du moins comme complémentaire en fonction du contexte dans lequel le requérant situe son action: s'il demande la mainlevée de la décision de soins sous contrainte, le juge judiciaire est seul compétent et pourra éventuellement se prononcer sur le respect des droits du patient; si le requérant conteste en revanche tel aspect du régime auquel il est soumis (transfert en UMD par exemple) sans pour autant demander la mainlevée de la décision administrative d'hospitalisation complète et en dehors du contrôle systématique du juge judiciaire, le juge administratif devrait pouvoir se prononcer ${ }^{108}$. Mais cette complémentarité, si elle existe, ne peut être que marginale et ne constitue pas une solution véritablement satisfaisante.

\section{B. Une nouvelle répartition?}

Les apories du système actuel imposent de réfléchir à une répartition effectivement soucieuse des droits des administrés et de l'intérêt général, plutôt qu'obsédée par le respect des frontières entre juridictions, comme ce fut le cas tout au long du $\mathrm{XX}^{\mathrm{e}}$ siècle. Plusieurs options sont dès lors envisageables si l'on souhaite l'intervention d'un juge à la fois indépendant, efficace et techniquement compétent: la concurrence entre les deux ordres de juridiction (1), la création d'un bloc de compétence au profit d'un seul ordre de juridiction (2).

\section{L'option de la concurrence}

Parmi les améliorations susceptibles d'être envisagées, l'une d'entre elles pourrait consister à mettre en place une compétence concurrente des deux ordres de juridiction: dès lors notamment qu'il y a urgence à intervenir, peu importe le juge saisi du moment qu'un juge doté de pouvoirs suffisants peut intervenir. Exclu par le juge administratif ${ }^{109}$ et apparemment par le législateur de 2011, ce schéma fut un moment retenu en cas de voie de fait puisque le juge administratif du référé-liberté a admis en janvier 2013 de faire cesser l'atteinte au droit de propriété constitutive en l'espèce d'une voie de fait ${ }^{110}$. Il n'est cependant pas certain que cette compétence concurrente subsiste après la redéfinition de la voie de fait par le Tribunal des conflits en juin $2013^{111}$. Ce dernier a en effet resserré la voie de fait sur les atteintes à la liberté individuelle (celle de l'article 66 vraisemblablement) et au cas d'extinction du droit de propriété. Cette nouvelle définition de la voie de fait est certes plus cohérente avec l'actuel libellé de l'article 66 de la Constitution, mais on pourrait en conclure que le Tribunal des conflits souhaite cantonner le juge administratif des référés aux atteintes graves et manifestement illégales portées par l'administration à toutes les libertés, sauf à la liberté individuelle dont la protection resterait confiée exclusivement au juge judiciaire. Comment justifier pourtant de renvoyer le plaideur devant un autre ordre de juridiction dès lors qu'il y a urgence et illégalité manifeste de l'atteinte à sa liberté individuelle?

En dehors de l'hypothèse d'une illégalité manifeste, on pourrait tout aussi bien concevoir cette concurrence de compétences. Dès lors qu'un juge est saisi (qu'il soit administratif ou judiciaire), il serait compétent pour traiter la totalité du litige. Cette option est aujourd'hui exclue pour les soins sans consentement, et le juge administratif la rejette de façon catégorique, y compris pour des contentieux relatifs aux programmes de soins ambulatoires ${ }^{112}$. Une telle répartition aurait pour inconvénient de risquer les contradictions de jurisprudence entre les deux ordres: l'un pourrait accepter plus facilement que l'autre la remise en liberté du patient. Cette option impliquerait en outre d'accroître les pouvoirs du juge administratif en lui permettant de statuer sur la légalité

106. Voir les art. L. 521-1 et 521-2 CJA.

107. Voir supra I.

108. Voir supra I, mais on a vu à quel point les solutions en la matière étaient incertaines.

109. Voir CE, ord., $1^{\text {er }}$ avril 2010, $\mathrm{n}^{\circ} 335753$, et R. Bonnefont, «Les limites du référé-liberté en matière d'hospitalisation d'office», Les petites affiches, $\mathrm{n}^{\circ}$ 150, 29 juillet 2010, p. 15: «la compétence du juge des référés ne s’étend pas au-delà de la compétence normale des juridictions administratives et le juge du référé-liberté du tribunal administratif de Nîmes a considéré ici qu'il ne pouvait en aucun cas se prononcer sur l'objet de la demande sans empiéter sur la compétence du juge judiciaire. Il a estimé qu'il ne pouvait pas enjoindre à l'administration de mettre fin à l'hospitalisation d'office sans se livrer à un contrôle matériel de la décision d'internement. / Selon l'article L. 3211-12 du Code de la santé publique, la mainlevée de l'arrêté d'hospitalisation d'office relève de la compétence du juge judiciaire et, plus précisément, du juge des libertés et de la détention du tribunal de grande instance. / Il ne paraît pas, en effet, raisonnablement envisageable de prononcer la fin de l'internement sans se prononcer sur le bien-fondé d'une telle mesure, c'est-à-dire sur sa nécessité au regard de l'état pathologique de la personne et du danger qu'elle représente pour la société et pour elle-même. / Mais, comme le remarquaient Claire Landais et Frédéric Lénica, ce pas avait, semble-t-il, déjà été franchi par le juge du référé-suspension (CE, 9 novembre 2001, $\mathrm{n}^{\circ}$ 235247, M. D., Lebon, p. 547). Le juge du référé-liberté allait-il, cette fois-ci, franchir le Rubicon afin de prouver que les mots ont un sens? Pas tout à fait car le Conseil d'État s'est arrêté au milieu du gué...».

110. CE, ord., Commune de Chirongui, 23 janvier 2013, n³ 365262, P. Delvolvé, "Référé-liberté et voie de fait», Revue française de droit administratif, $\mathrm{n}^{\circ}$ 2, 2013, p. 299-304; à la suite de cette ordonnance, le Tribunal des conflits a redéfini la notion de voie de fait de manière à la fois plus restrictive et plus cohérente au regard des fondements constitutionnels actuels (voir TC, 17 juin 2013, $\mathrm{n}^{\circ} \mathrm{C}_{3911}$ ).

111. TC, 17 juin 2013, $\mathrm{n}^{\circ}$ C3911, M.-C. de Montecler, «Le Tribunal des conflits redéfinit la voie de fait», L'actualité juridique. Droit administratif, $\mathrm{n}^{\circ}$ 22, 2013, p. 1245; X. Domino, A. Bretonneau, «La voie de fait mise au régime sec », L'actualité juridique. Droit administratif, n 27,2013, p. 1568-1571 P. Delvolvé, «Voie de fait: limitation et fondements », Revue française de droit administratif, n 5, 2013, p. 1041.

112. Voir CE, ord., 16 juillet 2012, $\mathrm{n}^{\circ} 360793$. 
des agissements de l'administration sans qu'il soit besoin d'invoquer l'urgence et l'illégalité manifeste. Aujourd'hui en effet, cette contestation n'est possible que dans le cadre d'une action en réparation ou dans celui du référé-liberté. Le recours pour excès de pouvoir est ainsi sans utilité pour contester par exemple le défaut d'information du patient sur ses droits lors d'une décision d'admission en soins sans consentement.

Cette solution se heurte surtout à l'intervention obligatoire du juge judiciaire sur le fondement de l'article 66 . Dès lors que le législateur est contraint de prévoir son intervention pour autoriser le maintien du patient en hospitalisation complète, il paraît difficile d'organiser une telle concurrence de compétences.

\section{L'option du bloc de compétence}

La solution consistant à confier la totalité du contentieux des soins sans consentement à un seul et même juge exclusivement compétent est à la fois plus simple et sans doute plus radicale. Elle nous paraît s'imposer tant pour éviter l'extrême complexité du contentieux des soins sans consentement que pour améliorer la protection des droits des individus en cas de restriction de leur droit à la sûreté. Reste à savoir à quel juge confier le contentieux.

Ce pourrait être le juge judiciaire dans une sorte de continuité des évolutions jurisprudentielles et législatives de ces dernières années; c'était d'ailleurs la solution préconisée par la Commission nationale consultative des droits de l'homme ${ }^{113}$ et par Jean-René Lecerf, auteur d'un amendement déposé au cours de la discussion de la loi du 5 juillet $2011^{114}$. Au vu de la situation actuelle, on peut penser que c'est plus ou moins la solution à laquelle les juridictions administratives souhaiteraient parvenir. Mais ce n'est pas forcément celle voulue par les juridictions judiciaires, du moins sans que leur soient accordés des moyens supplémentaires ${ }^{115}$. Cette solution se heurte en outre au principe de compétence réservée du juge administratif.
À l'inverse, l'unification du contentieux pourrait se faire au profit du juge administratif au titre de sa compétence technique pour statuer sur des décisions relevant du droit administratif. C'est cette solution que préconise Cécile Castaing après avoir mis en lumière les difficultés de la répartition des compétences issue de la réforme de 2011; elle estime notamment qu'il aurait été "plus logique, dans l'intérêt d'une bonne administration de la justice, de transférer le contentieux de la légalité interne des décisions administratives de soins psychiatriques privatives de liberté au juge administratif ${ }^{116}$. Cette solution ne permettrait pas cependant de régler complètement le problème si l'on ne remet pas en cause simultanément la rédaction de l'article 66. Tant que le juge judiciaire devra statuer sur la prolongation de l'hospitalisation du patient, et si l'on veut que son contrôle soit effectif, il faut lui donner tous les moyens de vérifier la légalité de la décision qui la fonde. Dès lors, s'il exerce ce contrôle de façon systématique dans les douze jours de la décision administrative d'admission en soins sans consentement, il serait peu cohérent de le déposséder des recours visant à la mainlevée de la décision ou à la réparation des dommages causés par une décision illégale.

L'habeas corpus exige l'intervention d'un juge indépendant et impartial pour sauvegarder la sûreté des administrés. Peu devrait importer le juge (qu'il soit judiciaire ou administratif); ce qui importe, en revanche, c'est qu'un juge impartial et techniquement compétent se prononce rapidement lorsque l'individu fait l'objet d'une détention, d'une rétention ou d'un internement. Mais l'article 66 est formulé de telle sorte que cette intervention ne peut être confiée qu'à l'autorité judiciaire; une « autorité judiciaire » composée, qui plus est, des magistrats du siège et de ceux du parquet ${ }^{117}$ dont on sait que la Cour européenne des droits de l'homme a relevé à plusieurs reprises le manque d'indépendance vis-à-vis de l'exécutif ${ }^{118}$.

Dès lors, ne faudrait-il pas réécrire l'article 66 et confier la garde de la liberté individuelle, non pas à l'autorité judiciaire, ni même au juge judiciaire, mais plus

113. Voir l'avis rendu le 31 mars 2011 par la Commission nationale consultative des droits de l'homme: «On pourrait aussi imaginer qu'il soit fait un bloc de compétences au profit du juge judiciaire, afin que celui-ci connaisse de l'intégralité du contentieux du soin psychiatrique contraint: la concurrence entre la compétence du juge administratif pour connaître des décisions du directeur de l'établissement et celle du juge judiciaire pour décider du maintien de l'hospitalisation sous contrainte ou de la mainlevée de celle-ci n'est guère satisfaisante».

114. Amendement $n^{\circ} 34$ présenté par J.-R. Lecerf au nom de la commission des lois : «Après l'article 5 , insérer un article additionnel ainsi rédigé: / Le titre I ${ }^{\text {er }}$ du livre II de la troisième partie du même code est complété par un chapitre VI ainsi rédigé : "Chapitre VI: Contentieux/ Art. L. 3216-1. - Le contentieux né de l'application du présent titre est exclusivement porté devant l’autorité judiciaire” ", compte rendu intégral des débats au Sénat, séance du 13 mai 2011.

115. Voir dans le même sens, A. Farinetti, «L'unification du contentieux...»; N. Albert, «Hospitalisation d'office: le juge judiciaire sous pression. À propos de la décision CEDH, 14 avril 2011, n 35079/o6, Patoux c/ France», La semaine juridique, édition administrations et collectivités territoriales, $\mathrm{n}^{\circ} 17,26$ avril 2011 , act. $\mathrm{n}^{\circ} 307$.

116. C. Castaing, «La volonté... », p. 153

117. CC, déc. $\mathrm{n}^{\circ}$ 93-326 DC du 11 août 1993, cons. 5, Loi modifiant la loi $n^{\circ}$ 93-2 du 4 janvier 1993 portant réforme du code de procédure pénale: «l'autorité judiciaire qui, en vertu de l'article 66 de la Constitution, assure le respect de la liberté individuelle, comprend à la fois les magistrats du siège et ceux du parquet».

118. Cour EDH, Medvedyev c. France, arrêt de chambre, 10 juillet 2008, n 3394/o3; Cour EDH, GC, Medvedyev c. France, 27 mars $2010, \mathrm{n}^{\circ} 3394 / 03$; Cour EDH, Moulin c. France, 23 novembre 2010, n³7104/06, \$ 57: «la Cour considère que, du fait de leur statut ainsi rappelé, les membres du ministère public, en France, ne remplissent pas l'exigence d'indépendance à l'égard de l'exécutif». 
simplement au juge, qu'il soit judiciaire ou administratif ${ }^{119}$ ? Une telle rédaction éviterait les difficultés actuelles visant à concilier la compétence judiciaire imposée en cas de restriction de la liberté individuelle et la compétence administrative liée à l'annulation des décisions de la puissance publique. Elle éviterait également les difficultés relatives au rôle des magistrats du parquet.

Si l'article 66 est reformulé de telle sorte que la protection de la liberté individuelle soit confiée au juge (et non plus à l'autorité judiciaire), le juge administratif serait compétent non seulement pour statuer sur les recours contre toutes les décisions administratives liées aux soins sans consentement, mais il le serait aussi en amont pour autoriser la prolongation de l'hospitalisation complète du patient. Il n'y aurait plus alors à s'interroger sur les frontières de compétence en aval, le juge administratif restant compétent pour statuer sur tout ce qui a trait aux décisions administratives prises en la matière. Cette réécriture de l'article 66 éviterait également les difficultés persistantes dans d'autres champs du contentieux, notamment dans celui des étrangers dont les droits sont trop souvent sacrifiés aux principes de répartition des compétences juridictionnelles.

Il est donc indispensable de concevoir la répartition à partir du respect des droits de l'individu et de renforcer à la fois l'indépendance et la culture des droits de l'homme dans les deux ordres de juridiction. C'est cette exigence qui fonde l'article 66 et qui n'est pas correctement satisfaite dans la rédaction actuelle de cette disposition, quels que soient les efforts éventuels des juges en la matière. Mais cette solution n'est vraisemblablement pas pour demain, puisqu'elle suppose au préalable une révision de la Constitution.

Au cours de ces vingt dernières années, le flux des recours n'a cessé d'augmenter devant chacun des ordres de juridiction, alors que dans le même temps les juges étaient soumis à une pression croissante pour rendre leurs jugements dans un délai raisonnable qui soit conforme aux exigences de la Cour européenne des droits de l'homme. Cette situation a conduit le législateur à multiplier les réformes de procédure; mais dans un contexte économique contraint, la plupart de ces réformes se sont faites à moyens constants. C'est notamment le cas de la loi de 2011 imposant au JLD un contrôle systématique des hospitalisations sans consentement. Même si les juges judiciaires ont fait preuve à cette occasion d'une très grande conscience professionnelle, il n'en reste pas moins que l'accroissement du contentieux en la matière pose des problèmes importants.

Il en va de même du côté du juge administratif confronté à la fois à des contentieux de masse et à la nécessité d'ouvrir plus largement son prétoire aux administrés dès lors que ces derniers invoquent la violation de leurs droits fondamentaux. La mise en place du référéliberté par la loi du 30 juin 2000 est en partie à l'origine d'un phénomène de vases communicants entre les deux ordres de juridiction, le succès de cette procédure ayant contribué à diminuer les recours devant le juge judiciaire. De même, l'inversion des priorités entre saisine du juge administratif et saisine du juge judiciaire en matière de rétention des étrangers a augmenté le nombre des recours portés devant le juge administratif et diminué les occasions de saisir le JLD compétent sur la prolongation de la rétention ${ }^{120}$.

À l'inverse de l'attitude qu'il adoptait au début du $\mathrm{XX}^{\mathrm{e}}$ siècle, le juge administratif n'est plus arcbouté sur la défense de son champ de compétence. Il cherche davantage à limiter la croissance exponentielle du contentieux et semble prêt à retenir une lecture extensive de la compétence du juge judiciaire sans que pour autant la ligne de partage entre les deux ordres de juridiction soit clairement fixée.

Outre une nouvelle clé de répartition du contentieux, la protection des droits du malade impose d'accroître les moyens des juridictions tant judiciaires qu'administratives. Qu'il s'agisse de l'augmentation du nombre des magistrats, des personnels administratifs ou des possibilités de recourir à l'expertise de psychiatres, ces réformes sont incontournables pour la mise en place d'un contrôle juridictionnel effectif ${ }^{121}$. Il en va de même d'un renforcement de l'indépendance des juges tant judiciaires qu'administratifs et de la nécessité d'insister dans leur formation sur les droits et libertés ainsi que sur les réalités concrètes vécues par les administrés les plus vulnérables.

119. Comparer la formulation adoptée par l'assemblée générale du Conseil d'État sur la proposition de Marcel Martin dans sa séance du 28 août 1958, Documents pour servir..., vol. III, p. 388: «L'autorité judiciaire est gardienne de la liberté individuelle. Nul ne peut être arbitrairement détenu. Les juridictions judiciaires et les juridictions administratives assurent, chacune en ce qui les concerne, le respect de ce principe dans les conditions prévues par la loi».

120. Loi du 16 juin 2011 réformant le CESEDA. On trouve une autre trace de ce phénomène de rééquilibrage avec la redéfinition de la voie de fait restreignant le champ de la compétence judiciaire (TC, 17 juin 2013, $\mathrm{n}^{\circ} \mathrm{C} 3911$ ).

121. Sur ces questions, voir notamment G. Lefrand, S. Blisko, Rapport d'information déposé par la commission des affaires sociales sur la mise en ceuvre de la loi $n^{\circ} 2011-803$ du 5 juillet 2011 relative aux droits et à la protection des personnes faisant l'objet de soins psychiatriques et aux modalités de leur prise en charge, n 4402 , enregistré à la présidence de l'Assemblée nationale le 22 février 2012. 